\title{
Maximum-likelihood Estimation for Diffusion Processes via Closed-form Density Expansions — Supplementary Material
}

\author{
Chenxu Li* \\ Department of Business Statistics and Econometrics \\ Guanghua School of Management \\ Email: cxli@gsm.pku.edu.cn
}

First Draft: October 18, 2010

This Version: April 6, 2013

\begin{abstract}
This supplementary material for Maximum-likelihood Estimation for Diffusion Processes via Closedform Density Expansions contains (1) closed-form formulas for $\Omega_{1}(y), \Omega_{2}(y)$ and $\Omega_{3}(y)$ (Section (1), (2) closed-form expansion formulas for the examples (Section 21), (3) detailed plots of errors for the examples (Section 3), (4) simulation results for the DMROU and SQR models (Section 4), (5) an alternative exhibition of the simulation results (Section [5), (6) two more examples for simulation study (Section [6), (7) a brief survey of the Malliavin Calculus and Watanabe-Yoshida Theory (Section 7), and (8) proof of Lemma 2 (Section 8 ).
\end{abstract}

\section{Closed-form Formulas for $\Omega_{1}(y), \Omega_{2}(y)$ and $\Omega_{3}(y)$}

Regardless of the dimension of diffusion processes, I concretely exemplify the closed-form expression (3.25) as well as the required conditional expectations by the first three correction terms. In the following formulas, $\delta_{i j}$ denotes the Kronecker delta symbol for a pair of indices $(i, j)$, which takes value 1 if $i=j$, and 0 otherwise.

For $k=1$, the first correction term is

$$
\begin{aligned}
\Omega_{1}(y)= & -\sum_{i=1}^{m} D_{i i}\left(x_{0}\right) \phi_{\Sigma\left(x_{0}\right)}(y)\left[-b_{i}\left(x_{0}\right)\left(\Sigma\left(x_{0}\right)^{-1} y\right)_{i}+\sum_{i_{1}=1, i_{2}=1}^{m} \sum_{l=1}^{m} \sigma_{l i_{2}}\left(x_{0}\right) \frac{\partial \sigma_{i i_{1}}}{\partial x_{l}}\left(x_{0}\right)\right. \\
& \left.\mathcal{D}_{i}\left(P_{\left(\left(i_{1}, i_{2}\right)\right)}\left(\sigma\left(x_{0}\right)^{-1} D\left(x_{0}\right)^{-1} y\right)\right)\right],
\end{aligned}
$$

where the polynomial $P_{\left(\left(i_{1}, i_{2}\right)\right)}$ as defined in (3.24) is explicitly given by

$$
P_{\left(\left(i_{1}, i_{2}\right)\right)}(z)=\frac{1}{2} z_{i_{1}} z_{i_{2}}
$$

${ }^{*}$ This work was initially supported in part by the faculty fellowship at Columbia University during the author's PhD study, and is now supported by the National Natural Science Foundation of China (Project 11201009), the Guanghua School of Management and the Center for Statistical Sciences at Peking University. 
For $k=2$, the second correction term is

$$
\begin{aligned}
& \Omega_{2}(y)=\frac{1}{2} \sum_{i, j=1}^{m} D_{i i}\left(x_{0}\right) D_{j j}\left(x_{0}\right) \phi_{\Sigma\left(x_{0}\right)}(y)\left[-b_{i}\left(x_{0}\right) b_{j}\left(x_{0}\right) \mathcal{D}_{j}\left(\left(\Sigma\left(x_{0}\right)^{-1} y\right)_{i}\right)\right. \\
& +b_{i}\left(x_{0}\right) \sum_{j_{1}, j_{2}=1}^{m}\left(\sum_{r=1}^{m} \sigma_{r j_{2}}\left(x_{0}\right) \frac{\partial \sigma_{j j_{1}}\left(x_{0}\right)}{\partial x_{r}}\right) \mathcal{D}_{j}\left(\mathcal{D}_{i}\left(P_{\left(\left(j_{1}, j_{2}\right)\right)}\left(\sigma\left(x_{0}\right)^{-1} D\left(x_{0}\right)^{-1} y\right)\right)\right) \\
& +b_{j}\left(x_{0}\right) \sum_{i_{1}, i_{2}=1}^{m}\left(\sum_{r=1}^{m} \sigma_{r i_{2}}\left(x_{0}\right) \frac{\partial \sigma_{i i_{1}}\left(x_{0}\right)}{\partial x_{r}}\right) \mathcal{D}_{j}\left(\mathcal{D}_{i}\left(P_{\left(\left(i_{1}, i_{2}\right)\right)}\left(\sigma\left(x_{0}\right)^{-1} D\left(x_{0}\right)^{-1} y\right)\right)\right) \\
& +\sum_{i_{1}, i_{2}, j_{1}, j_{2}=1}^{m}\left(\sum_{r=1}^{m} \sigma_{r i_{2}}\left(x_{0}\right) \frac{\partial \sigma_{i i_{1}}\left(x_{0}\right)}{\partial x_{r}}\right)\left(\sum_{r=1}^{m} \sigma_{r j_{2}}\left(x_{0}\right) \frac{\partial \sigma_{j j_{1}}\left(x_{0}\right)}{\partial x_{r}}\right) \\
& \text {. } \left.\mathcal{D}_{j}\left(\mathcal{D}_{i}\left(P_{\left(\left(i_{1}, i_{2}\right),\left(j_{1}, j_{2}\right)\right)}\left(\sigma\left(x_{0}\right)^{-1} D\left(x_{0}\right)^{-1} y\right)\right)\right)\right]-\sum_{i=1}^{m} D_{i i}\left(x_{0}\right) \phi_{\Sigma\left(x_{0}\right)}(y) \\
& \cdot\left[\sum_{i_{1}=1}^{m}\left(\sum_{r=1}^{m} b_{r}\left(x_{0}\right) \frac{\partial \sigma_{i i_{1}}\left(x_{0}\right)}{\partial x_{r}}\right) \mathcal{D}_{i}\left(P_{\left(\left(i_{1}, 0\right)\right)}\left(\sigma\left(x_{0}\right)^{-1} D\left(x_{0}\right)^{-1} y\right)\right)\right) \\
& +\sum_{i_{2}=1}^{m}\left(\sum_{r=1}^{m} \sigma_{r i_{2}}\left(x_{0}\right) \frac{\partial b_{i}\left(x_{0}\right)}{\partial x_{r}}\right) \mathcal{D}_{i}\left(P_{\left(\left(0, i_{2}\right)\right)}\left(\sigma\left(x_{0}\right)^{-1} D\left(x_{0}\right)^{-1} y\right)\right) \\
& \left.+\sum_{i_{1}, i_{2}, i_{3}=1}^{m}\left(\sum_{r_{1}, r_{2}=1}^{m} \sigma_{r_{2} i_{3}}\left(x_{0}\right) \sigma_{r_{1} i_{2}}\left(x_{0}\right) \frac{\partial^{2} \sigma_{i i_{1}}}{\partial x_{r_{2}} \partial x_{r_{1}}}\left(x_{0}\right)\right) \mathcal{D}_{i}\left(P_{\left(\left(i_{1}, i_{2}, i_{3}\right)\right)}\left(\sigma\left(x_{0}\right)^{-1} D\left(x_{0}\right)^{-1} y\right)\right)\right],
\end{aligned}
$$

where

$$
\begin{aligned}
P_{\left(\left(0, i_{2}\right)\right)}(z)= & \frac{1}{2} z_{i_{2}}, \\
P_{\left(\left(i_{1}, 0\right)\right)}(z)= & \frac{1}{2} z_{i_{1}} \\
P_{\left(\left(i_{1}, i_{2}, i_{3}\right)\right)}(z)= & \frac{1}{6} z_{i_{1}} z_{i_{2}} z_{i_{3}}+\frac{1}{12} \delta_{i_{1} i_{2}} z_{i_{3}}-\frac{1}{6} \delta_{i_{1} i_{3}} z_{i_{2}}+\frac{1}{12} \delta_{i_{2} i_{3}} z_{i_{1}}, \\
P_{\left(\left(i_{1}, i_{2}\right),\left(j_{1}, j_{2}\right)\right)}(z)= & \frac{1}{4} z_{j_{1}} z_{j_{2}} z_{i_{1}} z_{i_{2}}+\frac{1}{12} \delta_{i_{1} j_{1}} z_{j_{2}} z_{i_{2}}+\frac{1}{12} \delta_{i_{2} j_{2}} z_{j_{1}} z_{i_{1}}-\frac{1}{12} \delta_{i_{2} j_{1}} z_{j_{2}} z_{i_{1}}-\frac{1}{12} \delta_{i_{2} j_{1}} z_{j_{2}} z_{i_{1}} \\
& +\frac{1}{12} \delta_{i_{1} j_{1}} \delta_{i_{2} j_{2}}-\frac{1}{12} \delta_{i_{2} j_{1}} \delta_{i_{1} j_{2}} .
\end{aligned}
$$


For $k=3$, the third correction term is

$$
\begin{aligned}
& \Omega_{3}(y)=\sum_{i, j=1}^{m} D_{i i}\left(x_{0}\right) D_{j j}\left(x_{0}\right)\left[\sum_{\|\mathbf{i}\|=2,\|\mathbf{j}\|=3} C_{\mathbf{i}, i}\left(x_{0}\right) C_{\mathbf{j}, j}\left(x_{0}\right) \mathcal{D}_{j}\left(\mathcal{D}_{i}\left(P_{(\mathbf{i}, \mathbf{j})}\left(\sigma\left(x_{0}\right)^{-1} D\left(x_{0}\right)^{-1} y\right)\right)\right) \phi_{\Sigma\left(x_{0}\right)}(y)\right] \\
& -\sum_{i=1}^{m} D_{i i}\left(x_{0}\right)\left[\sum_{\|\mathbf{i}\|=4} C_{\mathbf{i}, i}\left(x_{0}\right) \mathcal{D}_{i}\left(P_{(\mathbf{i})}\left(\sigma\left(x_{0}\right)^{-1} D\left(x_{0}\right)^{-1} y\right)\right) \phi_{\Sigma\left(x_{0}\right)}(y)\right]-\frac{1}{6} \sum_{i, j, k=1}^{m} D_{i i}\left(x_{0}\right) D_{j j}\left(x_{0}\right) D_{k k}\left(x_{0}\right) \\
& \cdot\left[\sum_{\|\mathbf{i}\|=\|\mathbf{j}\|=\|\mathbf{k}\|=2} C_{\mathbf{i}, i}\left(x_{0}\right) C_{\mathbf{j}, j}\left(x_{0}\right) C_{\mathbf{k}, k}\left(x_{0}\right) \mathcal{D}_{k}\left(\mathcal{D}_{j}\left(\mathcal{D}_{i}\left(P_{(\mathbf{i}, \mathbf{j}, \mathbf{k})}\left(\sigma\left(x_{0}\right)^{-1} D\left(x_{0}\right)^{-1} y\right)\right)\right)\right) \phi_{\Sigma\left(x_{0}\right)}(y)\right] .
\end{aligned}
$$

In addition to (1.1) and (1.2), $\Omega_{3}(y)$ can be completed using

$$
\begin{aligned}
P_{\left(\left(0, i_{2}\right),\left(j_{1}, j_{2}\right)\right)}(z)= & \frac{1}{4} z_{i_{2}} z_{j_{1}} z_{j_{2}}-\frac{1}{12} \delta_{i_{2} j_{1}} z_{j_{2}}+\frac{1}{12} \delta_{i_{2} j_{2}} z_{j_{1}}, \\
P_{\left(\left(i_{1}, 0\right),\left(j_{1}, j_{2}\right)\right)}(z)= & \frac{1}{4} z_{i_{2}} z_{j_{1}} z_{j_{2}}-\frac{1}{12} \delta_{i_{1} j_{2}} z_{j_{1}}+\frac{1}{12} \delta_{i_{1} j_{1}} z_{j_{2}}, \\
P_{\left(\left(i_{1}, i_{2}, i_{3}\right),\left(j_{1}, j_{2}\right)\right)}(z)= & \frac{1}{12} z_{i_{1}} z_{i_{2}} z_{i_{3}} z_{j_{1}} z_{j_{2}}+\frac{1}{24} \delta_{i_{1} i_{2}} z_{i_{3}} z_{j_{1}} z_{j_{2}}-\frac{1}{12} \delta_{i_{1} i_{3}} z_{i_{2}} z_{j_{1}} z_{j_{2}}+\frac{1}{24} \delta_{i_{1} i_{3}} z_{i_{2}} z_{j_{1}} z_{j_{2}} \\
& +\frac{1}{24} \delta_{i_{1} j_{1}} z_{i_{2}} z_{i_{3}} z_{j_{2}}-\frac{1}{24} \delta_{i_{3} j_{1}} z_{i_{1}} z_{i_{2}} z_{j_{2}}-\frac{1}{24} \delta_{i_{1} j_{2}} z_{i_{2}} z_{i_{3}} z_{j_{1}}+\frac{1}{24} \delta_{i_{3} j_{2}} z_{i_{1}} z_{i_{2}} z_{j_{1}} \\
& +\frac{1}{24} \delta_{i_{1} j_{1}} \delta_{i_{2} j_{2}} z_{i_{3}}-\frac{1}{24} \delta_{i_{1} j_{2}} \delta_{i_{2} i_{1}} z_{i_{3}}+\frac{1}{24} \delta_{i_{2} j_{1}} \delta_{i_{2} j_{2}} z_{i_{1}}-\frac{1}{24} \delta_{i_{2} j_{2}} \delta_{i_{2} j_{1}} z_{i_{1}},
\end{aligned}
$$

and

$$
\begin{aligned}
P_{\left(\left(i_{1}, i_{2}, 0\right)\right)}(z)= & \frac{1}{6} z_{i_{1}} z_{i_{2}}+\frac{1}{12} \delta_{i_{1} i_{2}}, \\
P_{\left(\left(i_{1}, 0, i_{3}\right)\right)}(z)= & \frac{1}{6} z_{i_{1}} z_{i_{3}}-\frac{1}{6} \delta_{i_{1} i_{3}}, \\
P_{\left(\left(0, i_{2}, i_{3}\right)\right)}(z)= & \frac{1}{6} z_{i_{2}} z_{i_{3}}+\frac{1}{12} \delta_{i_{2} i_{3}}, \\
P_{\left(\left(i_{1}, i_{2}, i_{3}, i_{4}\right)\right)}(z)= & \frac{1}{24} z_{i_{1}} z_{i_{2}} z_{i_{3}} z_{i_{4}}+\frac{1}{24} \delta_{i_{1} i_{2}} z_{i_{3}} z_{i_{4}}+\frac{1}{24} \delta_{i_{2} i_{3}} z_{i_{1}} z_{i_{4}}+\frac{1}{24} \delta_{i_{3} i_{4}} z_{i_{1}} z_{i_{2}}-\frac{1}{24} \delta_{i_{1} i_{4}} z_{i_{2}} z_{i_{3}} \\
& -\frac{1}{24} \delta_{i_{1} i_{3}} z_{i_{2}} z_{i_{4}}-\frac{1}{24} \delta_{i_{2} i_{4}} z_{i_{1}} z_{i_{3}}+\frac{1}{24} \delta_{i_{1} i_{3}} \delta_{i_{2} i_{4}}-\frac{1}{24} \delta_{i_{1} i_{4}} \delta_{i_{2} i_{3}},
\end{aligned}
$$

moreover,

$$
\begin{aligned}
& P_{\left(\left(i_{1}, i_{2}\right),\left(j_{1}, j_{2}\right),\left(k_{1}, k_{2}\right)\right)}(z)=\frac{1}{8} z_{i_{1}} z_{i_{2}} z_{j_{1}} z_{j_{2}} z_{k_{1}} z_{k_{2}}+\frac{1}{24} \delta_{i_{1} j_{1}} z_{i_{2}} z_{j_{2}} z_{k_{1}} z_{k_{2}}+\frac{1}{24} \delta_{i_{2} j_{2}} z_{i_{1}} z_{j_{1}} z_{k_{1}} z_{k_{2}} \\
& -\frac{1}{24} \delta_{i_{2} j_{1}} z_{i_{1}} z_{j_{2}} z_{k_{1}} z_{k_{2}}-\frac{1}{24} \delta_{i_{1} j_{2}} z_{i_{2}} z_{j_{1}} z_{k_{1}} z_{k_{2}}+\frac{1}{24} \delta_{i_{1} k_{1}} z_{i_{2}} z_{j_{2}} z_{j_{1}} z_{k_{2}}+\frac{1}{24} \delta_{i_{2} k_{2}} z_{i_{1}} z_{j_{2}} z_{j_{1}} z_{k_{1}} \\
& -\frac{1}{24} \delta_{i_{2} k_{1}} z_{i_{1}} z_{j_{2}} z_{j_{1}} z_{k_{2}}-\frac{1}{24} \delta_{i_{1} k_{2}} z_{i_{2}} z_{j_{1}} z_{j_{2}} z_{k_{1}}+\frac{1}{24} \delta_{j_{1} k_{1}} z_{i_{1}} z_{i_{2 z}} z_{j_{2}} z_{k_{2}}+\frac{1}{24} \delta_{j_{2} k_{2}} z_{i_{1}} z_{i_{2}} z_{j_{1}} z_{k_{1}} \\
& -\frac{1}{24} \delta_{j_{2} k_{1}} z_{i_{1}} z_{i_{2}} z_{j_{1}} z_{k_{2}}-\frac{1}{24} \delta_{j_{1} k_{2}} z_{i_{1}} z_{i_{2}} z_{j_{2}} z_{k_{1}}+\frac{1}{24} \delta_{i_{1} k_{1}} \delta_{i_{2} k_{2}} z_{j_{1}} z_{j_{2}}-\frac{1}{24} \delta_{i_{2} k_{1}} \delta_{i_{1} k_{2}} z_{j_{1}} z_{j_{2}} \\
& +\frac{1}{24} \delta_{j_{1} k_{1}} \delta_{j_{2} k_{2}} z_{i_{1}} z_{i_{2}}-\frac{1}{24} \delta_{j_{2} k_{1}} \delta_{j_{1} k_{2}} z_{i_{1}} z_{i_{2}}+\frac{1}{24} \delta_{i_{1} j_{1}} \delta_{i_{2} j_{2}} z_{k_{1}} z_{k_{2}}-\frac{1}{24} \delta_{i_{1} j_{2}} \delta_{i_{2} j_{1}} z_{k_{1}} z_{k_{2}} .
\end{aligned}
$$




\section{Closed-form Expansion Formulas for the Examples}

In this section, I provide the first several expansion terms calculated from the general formula (3.25) for the models employed in Section 4 and 6. Higher order correction terms will be provided in the form of Mathematica notebook upon request.

\subsection{Model 1}

The MROU model:

$$
\begin{aligned}
& \Omega_{0}(y)=\frac{1}{\sqrt{2 \pi}} e^{-\frac{y^{2}}{2}}, \\
& \Omega_{1}(y)=\frac{1}{\sqrt{2 \pi}} e^{-\frac{y^{2}}{2}} \frac{y \kappa\left(\alpha-x_{0}\right)}{\sigma}, \\
& \Omega_{2}(y)=\frac{1}{\sqrt{2 \pi}} e^{-\frac{y^{2}}{2}}\left(\frac{y^{2} \kappa}{2 \sigma^{2}}\left(x_{0}^{2} \kappa-2 x_{0} \alpha \kappa+\alpha^{2} \kappa-\sigma^{2}\right)-\frac{\kappa}{2 \sigma^{2}}\left(x_{0}^{2} \kappa-2 x_{0} \alpha \kappa+\alpha^{2} \kappa-\sigma^{2}\right)\right), \\
& \Omega_{3}(y)=\frac{1}{\sqrt{2 \pi}} e^{-\frac{y^{2}}{2}}\left(-\frac{y^{3} \kappa^{2}\left(x_{0}-\alpha\right)}{6 \sigma^{3}}\left(x_{0}^{2} \kappa-2 x_{0} \alpha \kappa+\alpha^{2} \kappa-3 \sigma^{2}\right)-\frac{y \kappa^{2}\left(x_{0}-\alpha\right)}{6 \sigma^{3}}\left(-3 x_{0}^{2} \kappa+6 x_{0} \alpha \kappa-3 \alpha^{2} \kappa+6 \sigma^{2}\right)\right) .
\end{aligned}
$$

\section{$2.2 \quad$ Model 2}

The SQR model without Lamperti transform:

$$
\begin{aligned}
\Omega_{0}(y)= & \frac{1}{\sqrt{2 \pi}} e^{-\frac{y^{2}}{2}}, \\
\Omega_{1}(y)= & \frac{1}{\sqrt{2 \pi}} e^{-\frac{y^{2}}{2}}\left(\frac{y^{3} \sigma}{4 \sqrt{x_{0}}}+\frac{y}{4 \sqrt{x_{0}} \sigma}\left(-4 x_{0} \kappa+4 \alpha \kappa-3 \sigma^{2}\right)\right), \\
\Omega_{2}(y)= & \frac{1}{\sqrt{2 \pi}} e^{-\frac{y^{2}}{2}}\left(\frac{y^{2}}{32 x_{0} \sigma^{2}}\left(16 x_{0}^{2} \kappa^{2}-32 x_{0} \alpha \kappa^{2}+24 x_{0} \kappa \sigma^{2}+16 \alpha^{2} \kappa^{2}-40 \alpha \kappa \sigma^{2}+21 \sigma^{4}\right)\right. \\
& \left.+\frac{1}{32 x_{0} \sigma^{2}}\left(-16 x_{0}^{2} \kappa^{2}+32 x_{0} \alpha \kappa^{2}-16 \alpha^{2} \kappa^{2}+16 \alpha \kappa \sigma^{2}-3 \sigma^{4}\right)+\frac{y^{6} \sigma^{2}}{32 x_{0}}+\frac{y^{4}}{32 x_{0} \sigma^{2}}\left(-8 x_{0} \kappa \sigma^{2}+8 \alpha \kappa \sigma^{2}-11 \sigma^{4}\right)\right), \\
\Omega_{3}(y)= & \frac{1}{\sqrt{2 \pi}} e^{-\frac{y^{2}}{2}}\left(\frac{y^{9} \sigma^{3}}{384 x_{0}^{3 / 2}}+\frac{y^{7}}{384 x_{0}^{3 / 2} \sigma^{3}}\left(-12 x_{0} \kappa \sigma^{4}+12 \alpha \kappa \sigma^{4}-24 \sigma^{6}\right)+\frac{y^{5}}{384 x_{0}^{3 / 2} \sigma^{3}}\left(48 x_{0}^{2} \kappa^{2} \sigma^{2}-96 x_{0} \alpha \kappa^{2} \sigma^{2}+132 x_{0} \kappa \sigma^{4}\right.\right. \\
& \left.+48 \alpha^{2} \kappa^{2} \sigma^{2}-180 \alpha \kappa \sigma^{4}+150 \sigma^{6}\right)+\frac{y}{384 x_{0}^{3 / 2} \sigma^{3}}\left(-64 x_{0}^{3} \kappa^{3}+192 x_{0}^{2} \alpha \kappa^{3}-192 x_{0}^{2} \kappa^{2} \sigma^{2}-192 x_{0} \alpha^{2} \kappa^{3}+576 x_{0} \alpha \kappa^{2} \sigma^{2}\right. \\
& \left.-252 x_{0} \kappa \sigma^{4}+64 \alpha^{3} \kappa^{3}-384 \alpha^{2} \kappa^{2} \sigma^{2}+572 \alpha \kappa \sigma^{4}-240 \sigma^{6}\right)+\frac{y}{384 x_{0}^{3 / 2} \sigma^{3}}\left(192 x_{0}^{3} \kappa^{3}-576 x_{0}^{2} \alpha \kappa^{3}+48 x_{0}^{2} \kappa^{2} \sigma^{2}+576 x_{0} \alpha^{2} \kappa^{3}\right. \\
& \left.\left.-480 x_{0} \alpha \kappa^{2} \sigma^{2}+36 x_{0} \kappa \sigma^{4}-192 \alpha^{3} \kappa^{3}+432 \alpha^{2} \kappa^{2} \sigma^{2}-276 \alpha \kappa \sigma^{4}+45 \sigma^{6}\right)\right) .
\end{aligned}
$$

The SQR model after Lamperti transform:

$$
\begin{aligned}
\Omega_{0}(y)= & \frac{1}{\sqrt{2 \pi}} e^{-\frac{y^{2}}{2}} \\
\Omega_{1}(y)= & \frac{1}{\sqrt{2 \pi}} e^{-\frac{y^{2}}{2}} y\left(\frac{\lambda}{x_{0}}-\frac{\kappa x_{0}}{2}\right) \\
\Omega_{2}(y)= & \frac{1}{\sqrt{2 \pi}} e^{-\frac{y^{2}}{2}}\left(y^{2}-1\right) \frac{4(\lambda-1) \lambda+\kappa^{2} x_{0}^{4}-2 x_{0}^{2}(2 \kappa \lambda+\kappa)}{8 x_{0}^{2}} \\
\Omega_{3}(y)= & \frac{1}{\sqrt{2 \pi}} e^{-\frac{y^{2}}{2}}\left(y^{3} \frac{\left(8(\lambda-2)(\lambda-1) \lambda-\kappa^{3} x_{0}^{6}+6 \kappa^{2}(\lambda+1) x_{0}^{4}-12 \kappa \lambda^{2} x_{0}^{2}\right)}{48 x_{0}^{3}}\right. \\
& \left.+y \frac{\left(-24(\lambda-1) \lambda^{2}+24(\lambda-1) \lambda+3 \kappa^{3} x_{0}^{6}-18 \kappa^{2} \lambda x_{0}^{4}-12 \kappa^{2} x_{0}^{4}+36 \kappa \lambda^{2} x_{0}^{2}\right)}{48 x_{0}^{3}}\right),
\end{aligned}
$$


where $\lambda=\left(4 \kappa \alpha-\sigma^{2}\right) / 2 \sigma^{2}$.

\section{$2.3 \quad$ Model 3}

The MROU model:

$$
\begin{aligned}
\Omega_{0}\left(y_{1}, y_{2}\right)= & \frac{1}{2 \pi} e^{-\frac{y_{1}^{2}+y_{2}{ }^{2}}{2}}, \\
\Omega_{1}\left(y_{1}, y_{2}\right)= & \frac{1}{2 \pi} e^{-\frac{y_{1}^{2}+y_{2}^{2}}{2}}\left(y_{1}\left(-x_{10} \kappa_{11}+\alpha_{1} \kappa_{11}\right)+y_{2}\left(-x_{10} \kappa_{21}+\alpha_{1} \kappa_{21}+\left(-x_{20}+\alpha_{2}\right) \kappa_{22}\right)\right), \\
\Omega_{2}\left(y_{1}, y_{2}\right)= & \frac{1}{2 \pi} e^{-\frac{y_{1}^{2}+y_{2} 2}{2}}\left(\frac{1}{2} y_{1}^{2}\left(\kappa_{11}^{2}\left(x_{10}-\alpha_{1}\right)^{2}-\kappa_{11}\right)+\frac{1}{2} y_{2} y_{1}\left(2 \kappa_{11}\left(x_{10}-\alpha_{1}\right)\left(\kappa_{22}\left(x_{20}-\alpha_{2}\right)+x_{10} \kappa_{21}-\alpha_{1} \kappa_{21}\right)-\kappa_{21}\right)\right. \\
& +\frac{1}{2} y_{2}^{2}\left(2 \alpha_{1} \kappa_{22} \kappa_{21}\left(\alpha_{2}-x_{20}\right)-2 x_{10} \kappa_{21}\left(\kappa_{22}\left(\alpha_{2}-x_{20}\right)+\alpha_{1} \kappa_{21}\right)+\kappa_{22}\left(-2 x_{20} \alpha_{2} \kappa_{22}+x_{20}^{2} \kappa_{22}+\alpha_{2}^{2} \kappa_{22}-1\right)\right. \\
& \left.+x_{10}^{2} \kappa_{21}^{2}+\alpha_{1}^{2} \kappa_{21}^{2}\right)+\frac{1}{2}\left(\kappa_{11}^{2}\left(-\left(x_{10}-\alpha_{1}\right)^{2}\right)-2 \alpha_{1} \kappa_{21} \kappa_{22}\left(\alpha_{2}-x_{20}\right)-\kappa_{22}\left(-2 x_{20} \alpha_{2} \kappa_{22}+x_{20}^{2} \kappa_{22}+\alpha_{2}^{2} \kappa_{22}-1\right)\right. \\
& \left.\left.+2 x_{10} \kappa_{21}\left(\kappa_{22}\left(\alpha_{2}-x_{20}\right)+\alpha_{1} \kappa_{21}\right)-x_{10}^{2} \kappa_{21}^{2}-\alpha_{1}^{2} \kappa_{21}^{2}+\kappa_{11}\right)\right)
\end{aligned}
$$

and

$$
\begin{aligned}
& \Omega_{3}\left(y_{1}, y_{2}\right)=\frac{1}{2 \pi} e^{-\frac{y_{1}^{2}+y_{2}^{2}}{2}}\left(\frac{1}{6} y_{1}^{3}\left(-x_{10}^{3} \kappa_{11}^{3}+3 x_{10}^{2} \alpha_{1} \kappa_{11}^{3}+\alpha_{1} \kappa_{11}^{2}\left(-3+\alpha_{1}^{2} \kappa_{11}\right)-3 x_{10} \kappa_{11}^{2}\left(-1+\alpha_{1}^{2} \kappa_{11}\right)\right)+\frac{1}{6} y_{2}\left(-3 x_{10} \kappa_{11} \kappa_{21}\right.\right. \\
& +3 x_{10}^{3} \kappa_{11}^{2} \kappa_{21}-9 x_{10}^{2} \alpha_{1} \kappa_{11}^{2} \kappa_{21}+3 x_{10}^{3} \kappa_{21}^{3}-9 x_{10}^{2} \alpha_{1} \kappa_{21}^{3}+9 x_{10} \alpha_{1}^{2} \kappa_{21}^{3}+3 x_{10}^{2}\left(x_{20}-\alpha_{2}\right) \kappa_{11}^{2} \kappa_{22}-6 x_{10} \kappa_{21} \kappa_{22}+9 x_{10}^{2}\left(x_{20}\right. \\
& \left.-\alpha_{2}\right) \kappa_{21}^{2} \kappa_{22}-18 x_{10} \alpha_{1}\left(x_{20}-\alpha_{2}\right) \kappa_{21}^{2} \kappa_{22}+9 x_{10} x_{20}^{2} \kappa_{21} \kappa_{22}^{2}-18 x_{10} x_{20} \alpha_{2} \kappa_{21} \kappa_{22}^{2}+9 x_{10} \alpha_{2}^{2} \kappa_{21} \kappa_{22}^{2}-3 x_{10} \alpha_{1} \kappa_{11}^{2} \\
& \cdot\left(-3 \alpha_{1} \kappa_{21}+2\left(x_{20}-\alpha_{2}\right) \kappa_{22}\right)+3 \kappa_{11}\left(\alpha_{1} \kappa_{21}+\left(-x_{20}+\alpha_{2}\right) \kappa_{22}\right)-3 \alpha_{1}^{2} \kappa_{11}^{2}\left(\alpha_{1} \kappa_{21}+\left(-x_{20}+\alpha_{2}\right) \kappa_{22}\right)-3 \alpha_{1}^{2} \kappa_{21}^{2} \\
& \cdot\left(\alpha_{1} \kappa_{21}+\left(-x_{20}+\alpha_{2}\right) \kappa_{22}\right)+6 \kappa_{22}\left(\alpha_{1} \kappa_{21}+\left(-x_{20}+\alpha_{2}\right) \kappa_{22}\right)+6 \alpha_{1}\left(x_{20}-\alpha_{2}\right) \kappa_{21} \kappa_{22}\left(\alpha_{1} \kappa_{21}+\left(-x_{20}+\alpha_{2}\right) \kappa_{22}\right) \\
& \left.-3 x_{20}^{2} \kappa_{22}^{2}\left(\alpha_{1} \kappa_{21}+\left(-x_{20}+\alpha_{2}\right) \kappa_{22}\right)+6 x_{20} \alpha_{2} \kappa_{22}^{2}\left(\alpha_{1} \kappa_{21}+\left(-x_{20}+\alpha_{2}\right) \kappa_{22}\right)-3 \alpha_{2}^{2} \kappa_{22}^{2}\left(\alpha_{1} \kappa_{21}+\left(-x_{20}+\alpha_{2}\right) \kappa_{22}\right)\right) \\
& +\frac{1}{6} y_{2}^{3}\left(-x_{10}^{3} \kappa_{21}^{3}+3 x_{10}^{2} \alpha_{1} \kappa_{21}^{3}-3 x_{10} \alpha_{1}^{2} \kappa_{21}^{3}-3 x_{10}^{2}\left(x_{20}-\alpha_{2}\right) \kappa_{21}^{2} \kappa_{22}+6 x_{10} \alpha_{1}\left(x_{20}-\alpha_{2}\right) \kappa_{21}^{2} \kappa_{22}-3 x_{10} \kappa_{21} \kappa_{22}(-1\right. \\
& \left.+x_{20}^{2} \kappa_{22}-2 x_{20} \alpha_{2} \kappa_{22}+\alpha_{2}^{2} \kappa_{22}\right)+\alpha_{1}^{2} \kappa_{21}^{2}\left(\alpha_{1} \kappa_{21}+\left(-x_{20}+\alpha_{2}\right) \kappa_{22}\right)-2 \alpha_{1}\left(x_{20}-\alpha_{2}\right) \kappa_{21} \kappa_{22}\left(\alpha_{1} \kappa_{21}+\left(-x_{20}+\alpha_{2}\right) \kappa_{22}\right) \\
& \left.+\kappa_{22}\left(-3+x_{20}^{2} \kappa_{22}-2 x_{20} \alpha_{2} \kappa_{22}+\alpha_{2}^{2} \kappa_{22}\right)\left(\alpha_{1} \kappa_{21}+\left(-x_{20}+\alpha_{2}\right) \kappa_{22}\right)\right)+\frac{1}{6} y_{1}^{2} y_{2}\left(-3 x_{10}^{3} \kappa_{11}^{2} \kappa_{21}+3 x_{10}^{2} \kappa_{11}^{2}\left(3 \alpha_{1} \kappa_{21}\right.\right. \\
& \left.+\left(-x_{20}+\alpha_{2}\right) \kappa_{22}\right)-3 x_{10} \kappa_{11}\left(\left(-2+3 \alpha_{1}^{2} \kappa_{11}\right) \kappa_{21}+2 \alpha_{1}\left(-x_{20}+\alpha_{2}\right) \kappa_{11} \kappa_{22}\right)+3 \kappa_{11}\left(-2 \alpha_{1} \kappa_{21}+\alpha_{1}^{3} \kappa_{11} \kappa_{21}+\left(x_{20}\right.\right. \\
& \left.\left.-\alpha_{2}\right) \kappa_{22}+\alpha_{1}^{2}\left(-x_{20}+\alpha_{2}\right) \kappa_{11} \kappa_{22}\right)+y_{1}\left(\frac { 1 } { 6 } y _ { 2 } ^ { 2 } \left(3 x_{10} \kappa_{21}^{2}-3 \alpha_{1} \kappa_{21}^{2}-3 x_{10}^{3} \kappa_{11} \kappa_{21}^{2}+9 x_{10}^{2} \alpha_{1} \kappa_{11} \kappa_{21}^{2}+3 \alpha_{1}^{3} \kappa_{11} \kappa_{21}^{2}\right.\right. \\
& +3\left(x_{20}-\alpha_{2}\right) \kappa_{21} \kappa_{22}-6 x_{10}^{2}\left(x_{20}-\alpha_{2}\right) \kappa_{11} \kappa_{21} \kappa_{22}-6 \alpha_{1}^{2}\left(x_{20}-\alpha_{2}\right) \kappa_{11} \kappa_{21} \kappa_{22}+3 \alpha_{1} \kappa_{11} \kappa_{22}\left(-1+x_{20}^{2} \kappa_{22}-2 x_{20} \alpha_{2} \kappa_{22}+\alpha_{2}^{2} \kappa_{22}\right) \\
& \left.-3 x_{10} \kappa_{11}\left(3 \alpha_{1}^{2} \kappa_{21}^{2}+4 \alpha_{1}\left(-x_{20}+\alpha_{2}\right) \kappa_{21} \kappa_{22}+\kappa_{22}\left(-1+x_{20}^{2} \kappa_{22}-2 x_{20} \alpha_{2} \kappa_{22}+\alpha_{2}^{2} \kappa_{22}\right)\right)\right)+\frac{1}{6}\left(-6 x_{10} \kappa_{11}^{2}+6 \alpha_{1} \kappa_{11}^{2}\right. \\
& +3 x_{10}^{3} \kappa_{11}^{3}-9 x_{10}^{2} \alpha_{1} \kappa_{11}^{3}+9 x_{10} \alpha_{1}^{2} \kappa_{11}^{3}-3 \alpha_{1}^{3} \kappa_{11}^{3}-3 x_{10} \kappa_{21}^{2}+3 \alpha_{1} \kappa_{21}^{2}+3 x_{10}^{3} \kappa_{11} \kappa_{21}^{2}-9 x_{10}^{2} \alpha_{1} \kappa_{11} \kappa_{21}^{2}-3 \alpha_{1}^{3} \kappa_{11} \kappa_{21}^{2} \\
& -3 x_{20} \kappa_{21} \kappa_{22}+3 \alpha_{2} \kappa_{21} \kappa_{22}+6 x_{10}^{2}\left(x_{20}-\alpha_{2}\right) \kappa_{11} \kappa_{21} \kappa_{22}+6 \alpha_{1}^{2}\left(x_{20}-\alpha_{2}\right) \kappa_{11} \kappa_{21} \kappa_{22}-3 \alpha_{1} \kappa_{11} \kappa_{22}\left(-1+x_{20}^{2} \kappa_{22}\right. \\
& \left.\left.\left.\left.\left.-2 x_{20} \alpha_{2} \kappa_{22}+\alpha_{2}^{2} \kappa_{22}\right)+3 x_{10} \kappa_{11}\left(3 \alpha_{1}^{2} \kappa_{21}^{2}+4 \alpha_{1}\left(-x_{20}+\alpha_{2}\right) \kappa_{21} \kappa_{22}+\kappa_{22}\left(-1+x_{20}^{2} \kappa_{22}-2 x_{20} \alpha_{2} \kappa_{22}+\alpha_{2}^{2} \kappa_{22}\right)\right)\right)\right)\right)\right) \text {. }
\end{aligned}
$$

\section{Detailed Performance of the Expansions for the Examples}

In Figures 1, 2] 3] and 4] I document detailed performance of the density approximation for the MROU, SQR (for both the direct expansion and the accelerated approach via Lamperti transform) and DMROU 
models, respectively. For the former two one-dimensional cases, i.e. the MROU and SQR models, I plot the errors of approximation corresponding to weekly monitoring frequency and orders ranging from $J=1,2, \cdots, 6$. For the latter set of graphs, I plot the contours of the approximation errors for the DMROU model corresponding to weekly monitoring frequency and orders ranging from $J=1,2, \cdots, 6$.

\section{Simulation Results for the DMROU and the SQR Models}

The following tables exhibit the Monte Carlo simulation results for the DMROU and the SQR models discussed in Section 6.

Table 1: Monte Carlo Evidence for the DMROU Model

Notes. The number of simulation trials is $N=1000$ and the number of observations on each path is $n=1000$.

\begin{tabular}{|c|c|c|c|c|c|c|c|c|}
\hline \multirow[t]{2}{*}{$\begin{array}{c}\text { Parameters } \\
\theta^{\text {True }}\end{array}$} & \multicolumn{2}{|c|}{$\begin{array}{l}\text { Asymptotic } \\
\widehat{\theta}_{n}-\theta^{\text {True }}\end{array}$} & \multicolumn{2}{|c|}{$\begin{array}{c}\text { Finite sample } \\
\widehat{\theta}_{n}-\theta^{\text {True }}\end{array}$} & \multicolumn{2}{|c|}{$\begin{array}{c}\text { Finite sample } \\
\widehat{\theta}_{n}^{(2)}-\widehat{\theta}_{n}\end{array}$} & \multicolumn{2}{|c|}{$\begin{array}{l}\text { Finite sample } \\
\widehat{\theta}_{n}^{(4)}-\widehat{\theta}_{n}\end{array}$} \\
\hline & Mean & Stddev & Mean & Stddev & Mean & Stddev & Mean & Stddev \\
\hline \multicolumn{9}{|l|}{$\Delta=1 / 252$} \\
\hline$\alpha_{1}=0$ & 0 & 0.100401 & -0.001686 & 0.097716 & 0.001444 & 0.028145 & 0.000709 & 0.023114 \\
\hline$\alpha_{2}=0$ & 0 & 0.051197 & -0.000560 & 0.049661 & 0.000178 & 0.008512 & 0.000070 & 0.008426 \\
\hline$\kappa_{11}=5$ & 0 & 1.587554 & 1.133810 & 2.013933 & 0.004577 & 0.208237 & 0.003392 & 0.167915 \\
\hline$\kappa_{21}=1$ & 0 & 1.602581 & 0.034982 & 2.019396 & -0.010490 & 0.098894 & 0.003190 & 0.096604 \\
\hline$\kappa_{22}=10$ & 0 & 2.240703 & 1.348146 & 2.581314 & 0.002389 & 0.053998 & -0.002482 & 0.057265 \\
\hline \multirow[t]{2}{*}{$\begin{array}{c}\text { Parameters } \\
\theta^{\text {True }}\end{array}$} & \multicolumn{2}{|c|}{$\begin{array}{l}\text { Asymptotic } \\
\widehat{\theta}_{n}-\theta^{\text {True }} \\
\end{array}$} & \multicolumn{2}{|c|}{$\begin{array}{l}\text { Finite sample } \\
\widehat{\theta}_{n}-\theta^{\text {True }}\end{array}$} & \multicolumn{2}{|c|}{$\begin{array}{c}\text { Finite sample } \\
\widehat{\theta}_{n}^{(3)}-\widehat{\theta}_{n}\end{array}$} & \multicolumn{2}{|c|}{$\begin{array}{c}\text { Finite sample } \\
\widehat{\theta}_{n}^{(6)}-\widehat{\theta}_{n}\end{array}$} \\
\hline & Mean & Stddev & Mean & Stddev & Mean & Stddev & Mean & Stddev \\
\hline \multicolumn{9}{|l|}{$\Delta=1 / 52$} \\
\hline$\alpha_{1}=0$ & 0 & 0.045625 & -0.000527 & 0.044492 & 0.000093 & 0.013668 & 0.000512 & 0.012092 \\
\hline$\alpha_{2}=0$ & 0 & 0.023290 & -0.000200 & 0.022371 & 0.000024 & 0.003641 & 0.000212 & 0.003543 \\
\hline$\kappa_{11}=5$ & 0 & 0.722188 & 0.247767 & 0.770279 & 0.577924 & 0.179183 & 0.000775 & 0.027134 \\
\hline$\kappa_{21}=1$ & 0 & 0.743389 & -0.012143 & 0.799808 & 0.210921 & 0.193220 & 0.002025 & 0.020130 \\
\hline$\kappa_{22}=10$ & 0 & 1.023690 & 0.266464 & 1.074969 & 2.294890 & 0.507876 & 0.004959 & 0.013778 \\
\hline \multicolumn{9}{|l|}{$\Delta=1 / 12$} \\
\hline$\alpha_{1}=0$ & 0 & 0.022066 & -0.000191 & 0.021364 & -0.000332 & 0.009245 & 0.000069 & 0.006972 \\
\hline$\alpha_{2}=0$ & 0 & 0.011475 & 0.000004 & 0.011109 & -0.000126 & 0.001862 & -0.000105 & 0.001783 \\
\hline$\kappa_{11}=5$ & 0 & 0.355289 & 0.053701 & 0.363768 & 2.681771 & 0.456786 & 0.022532 & 0.012014 \\
\hline$\kappa_{21}=1$ & 0 & 0.398388 & -0.015752 & 0.417193 & 1.356883 & 0.695915 & 0.069857 & 0.037665 \\
\hline$\kappa_{22}=10$ & 0 & 0.534837 & 0.066419 & 0.548926 & 13.523144 & 2.011232 & 0.041282 & 0.105874 \\
\hline
\end{tabular}




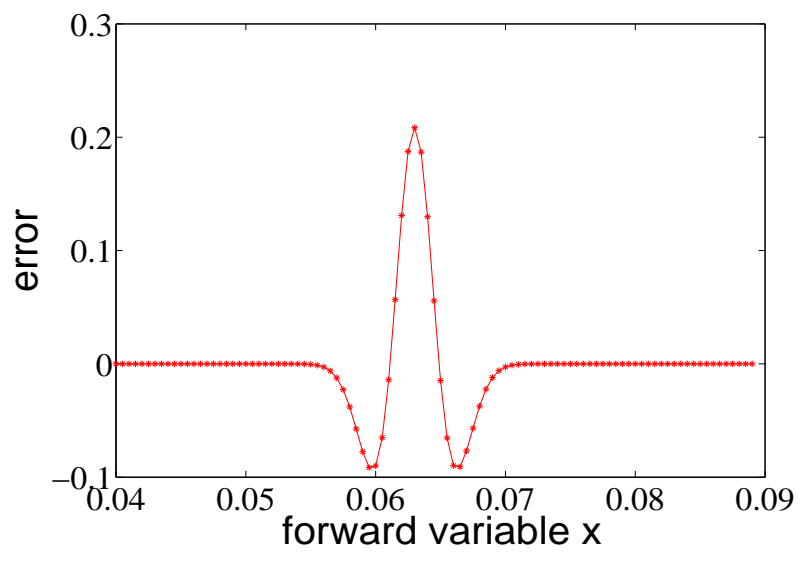

(a) $e^{(1)}$

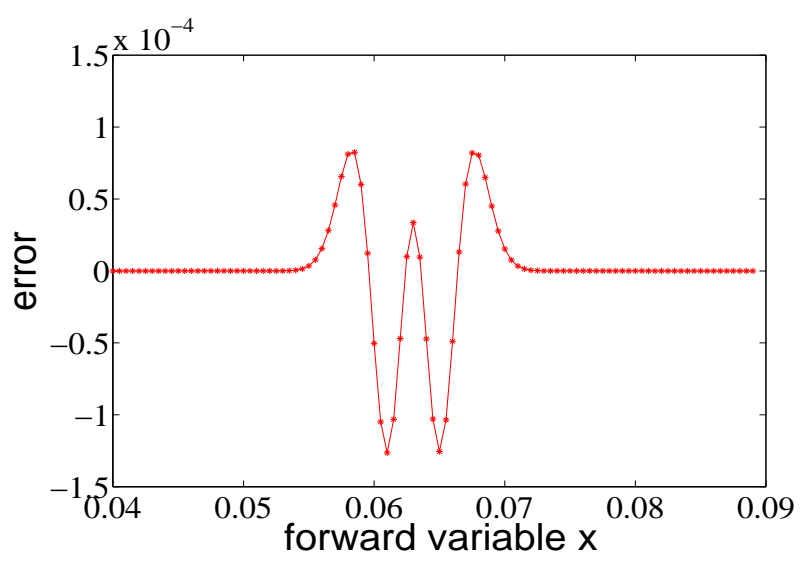

(c) $e^{(3)}$

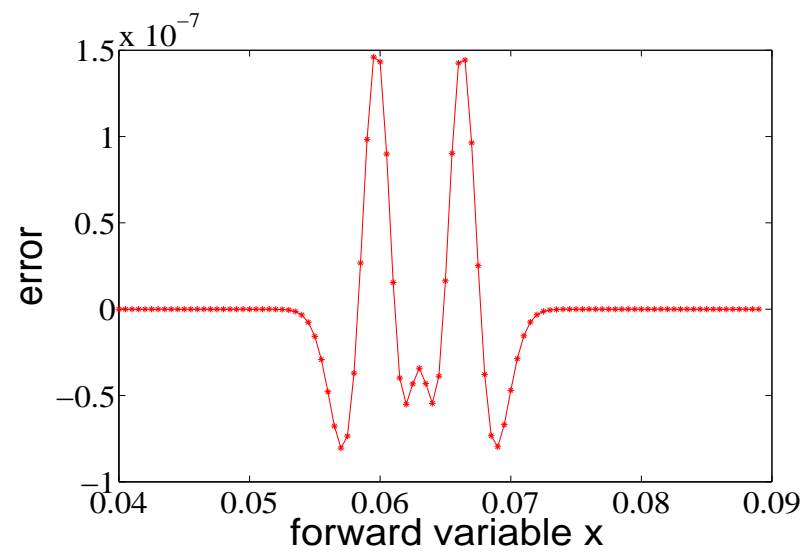

(e) $e^{(5)}$

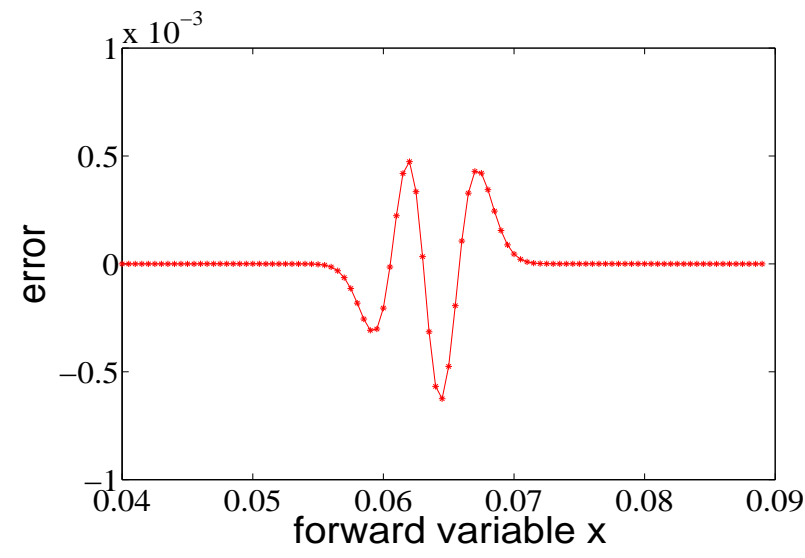

(b) $e^{(2)}$



(d) $e^{(4)}$



(f) $e^{(6)}$

Figure 1: Errors of Density Approximation for the Mean-reverting Ornstein-Uhlenbeck (MROU) Model, i.e. $e_{X}^{(J)}\left(\Delta, x \mid x_{0} ; \theta\right)$, for $J=1,2,3, \ldots, 6$ and $\Delta=1 / 52$. 




(a) $e^{(1)}$

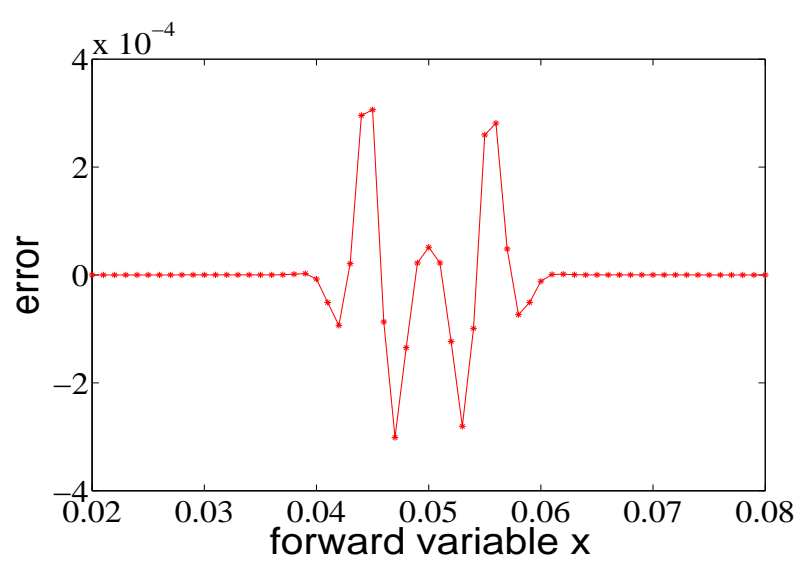

(c) $e^{(3)}$



(e) $e^{(5)}$

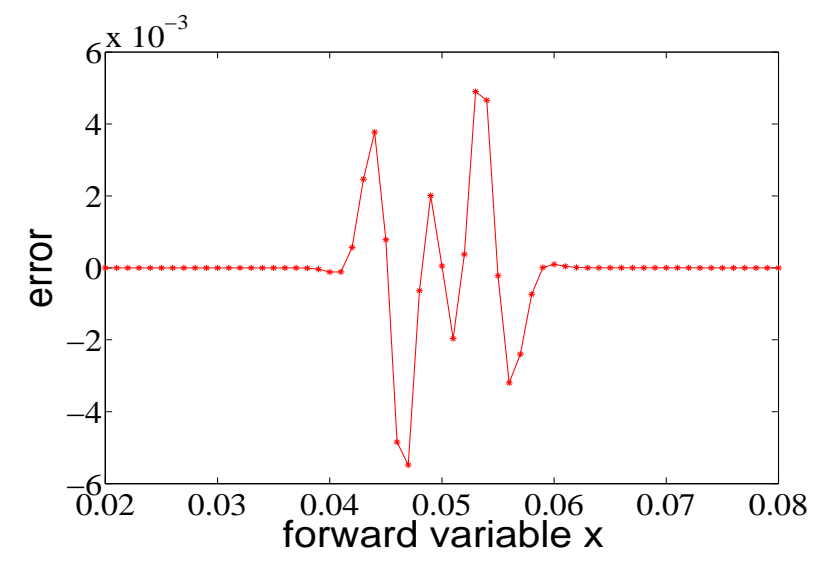

(b) $e^{(2)}$

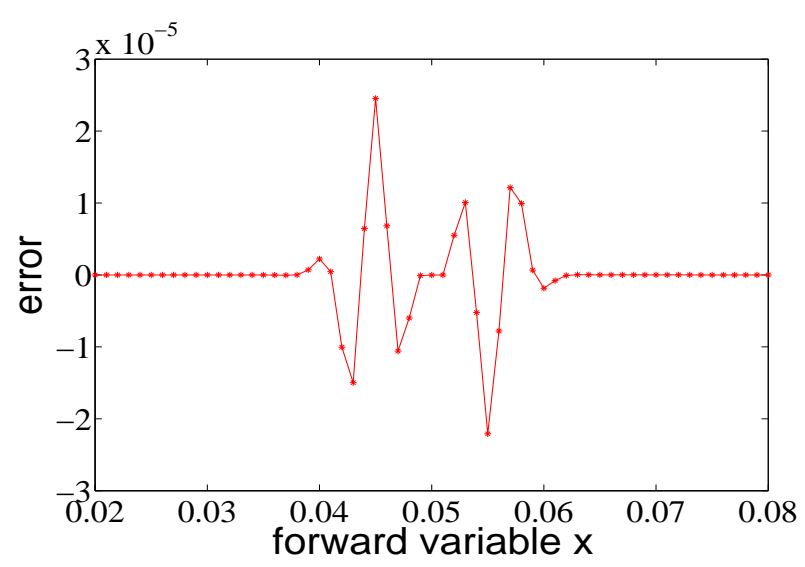

(d) $e^{(4)}$

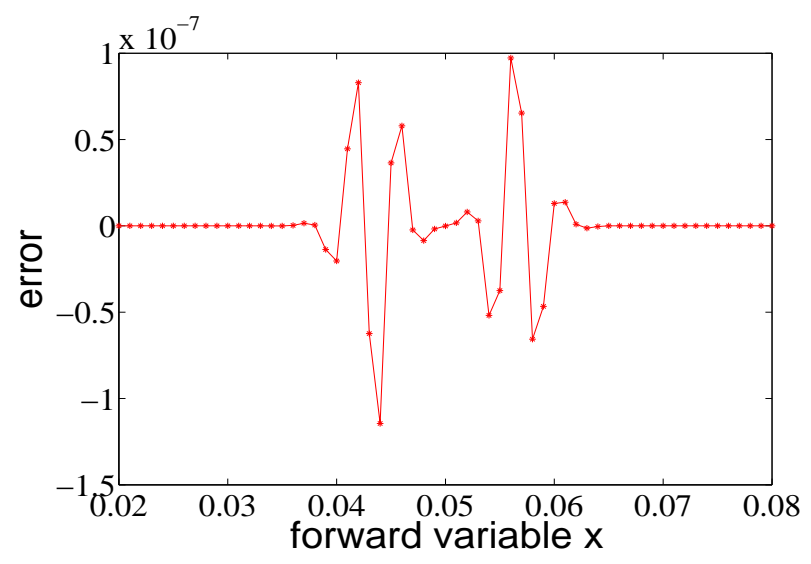

(f) $e^{(6)}$

Figure 2: Errors of Direct Approximation (according to (3.21)) for Feller's Square Root Diffusion (SQR) Model, i.e. $e_{X}^{(J)}\left(\Delta, x \mid x_{0} ; \theta\right)$, for $J=1,2,3, \ldots, 6$ and $\Delta=1 / 52$. 


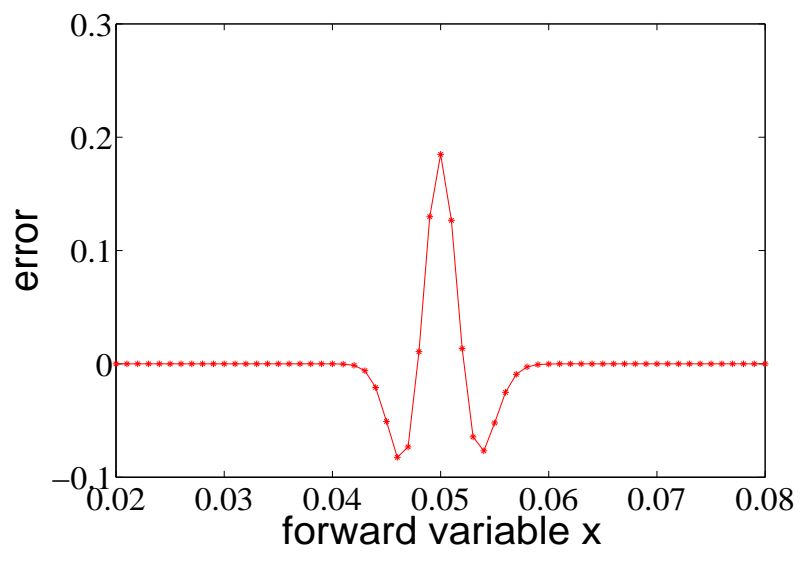

(a) $e^{(1)}$

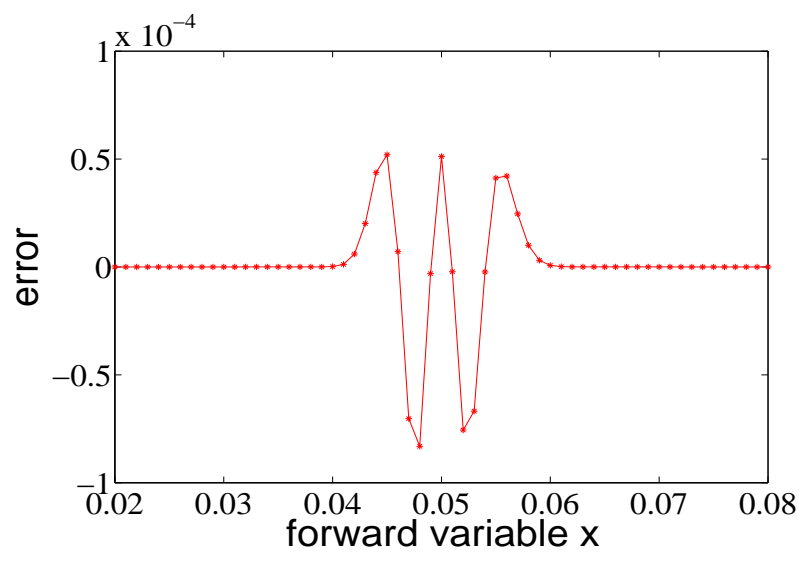

(c) $e^{(3)}$

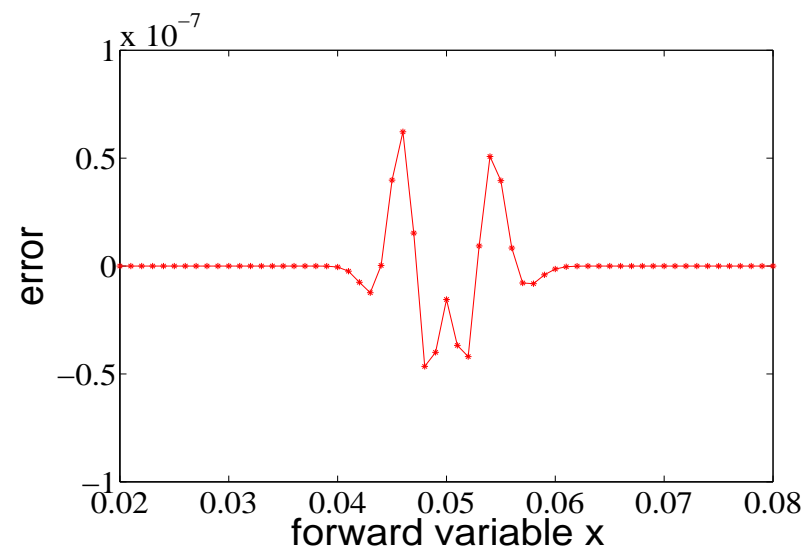

(e) $e^{(5)}$

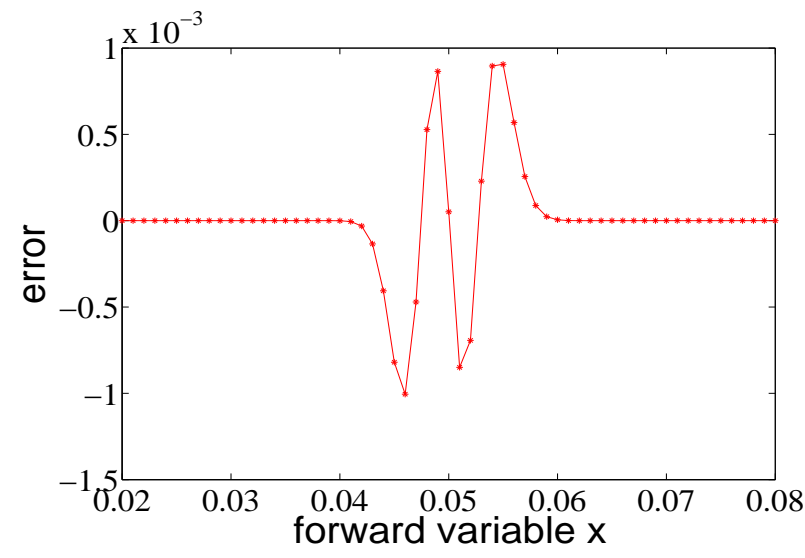

(b) $e^{(2)}$



(d) $e^{(4)}$



(f) $e^{(6)}$

Figure 3: Errors of Accelerated Approximation via Lamperti Transform for Feller's Square Root Diffusion (SQR) Model, i.e. $e_{X}^{(J)}\left(\Delta, x \mid x_{0} ; \theta\right)$, for $J=1,2,3, \ldots, 6$ and $\Delta=1 / 52$. 




(a) Contours of $\left|e^{(1)}\right|$

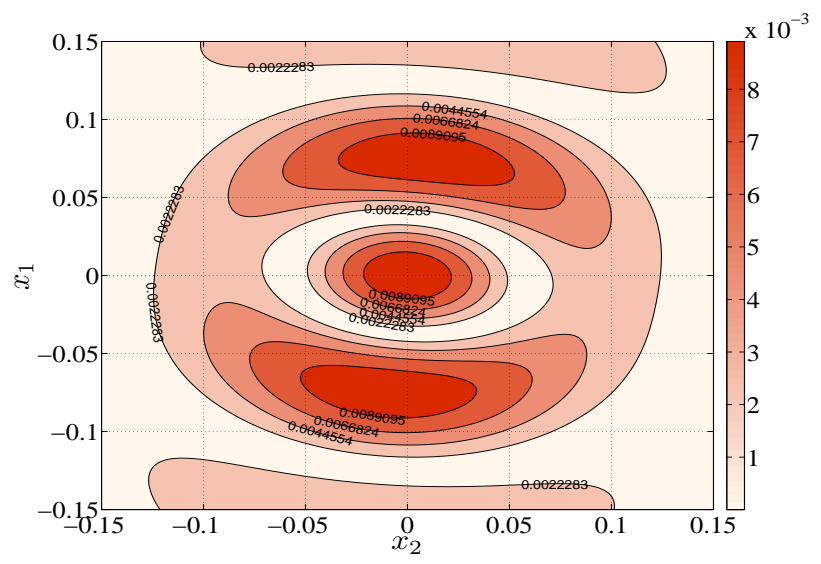

(c) Contours of $\left|e^{(3)}\right|$

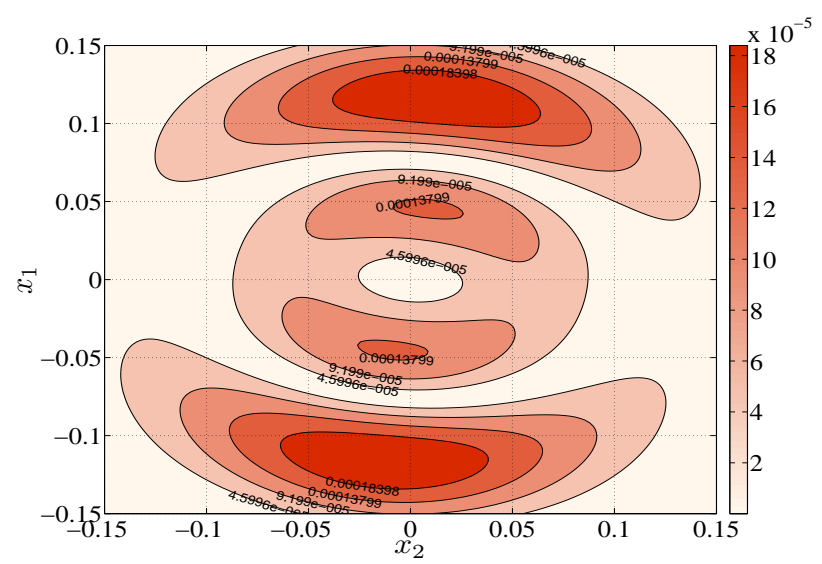

(e) Contours of $\left|e^{(5)}\right|$

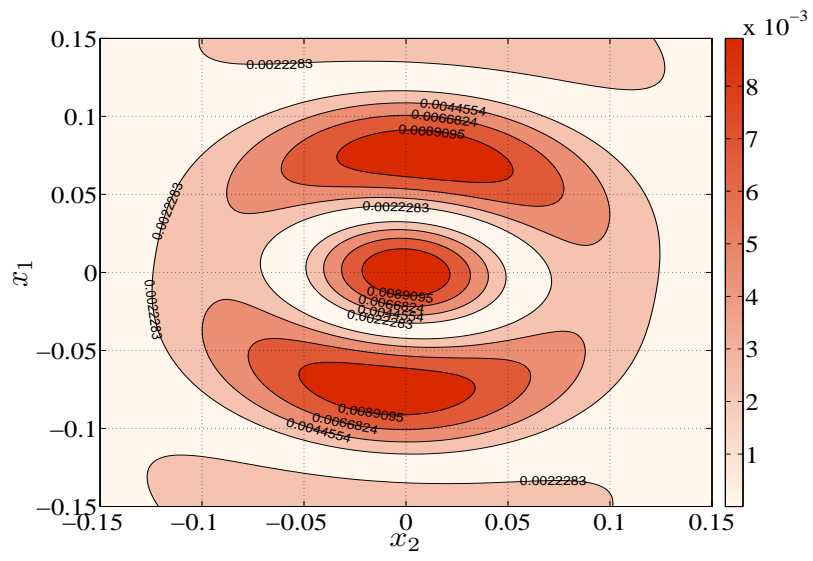

(b) Contours of $\left|e^{(2)}\right|$

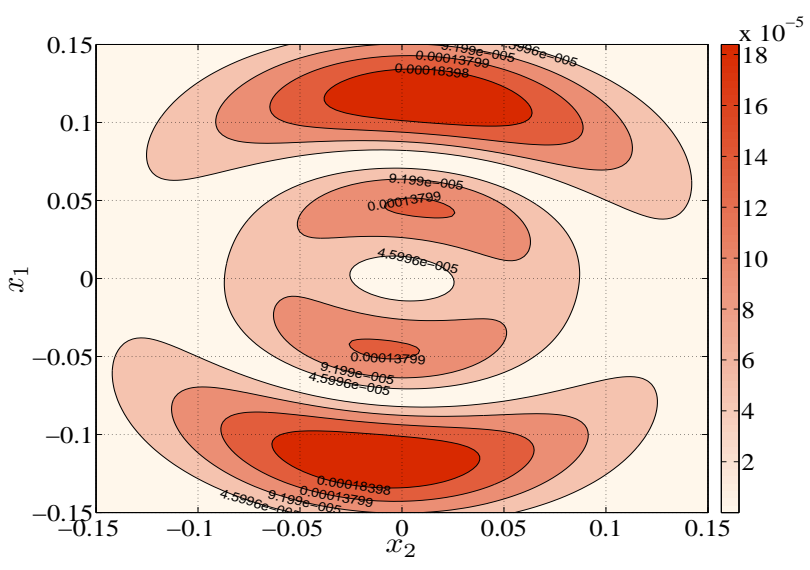

(d) Contours of $\left|e^{(4)}\right|$

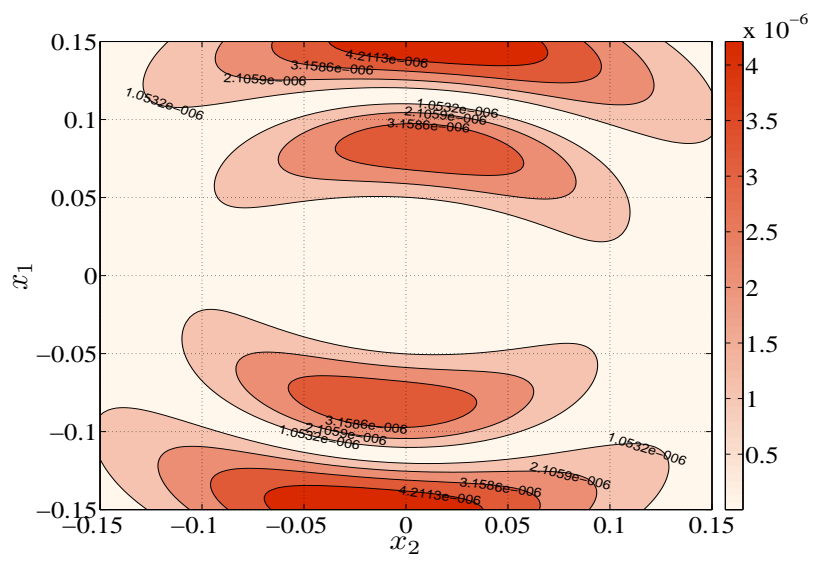

(f) Contours of $\left|e^{(6)}\right|$

Figure 4: Absolute Errors of Density Approximation for the Double Mean-reverting Ornstein-Uhlenbeck (DMROU) Model, i.e. contours of $\left|e_{X}^{(J)}\left(\Delta, x \mid x_{0} ; \theta\right)\right|$, for $J=1,2,3, \ldots, 6$ and $\Delta=1 / 52$. 


\section{Table 2: Monte Carlo Evidence for the SQR Model}

Notes. The number of simulation trials is $N=5000$ and the number of observations on each path is $n=1000$. The accelerated expansion refers to the combination of Lamperti transform and our main expansion procedure, while the direct expansion employs our main expansion procedure bypassing the Lamperti transform.

\begin{tabular}{|c|c|c|c|c|c|c|}
\hline \multirow[t]{2}{*}{$\begin{array}{c}\text { Parameters } \\
\theta^{\text {True }}\end{array}$} & \multicolumn{2}{|c|}{$\begin{array}{l}\text { Finite sample } \\
\widehat{\theta}_{n}-\theta^{\text {True }}\end{array}$} & \multicolumn{2}{|c|}{$\begin{array}{l}\text { Finite sample } \\
\widehat{\theta}_{n}^{(3)}-\widehat{\theta}_{n}\end{array}$} & \multicolumn{2}{|c|}{$\begin{array}{c}\text { Finite sample } \\
\widehat{\theta}_{n}^{(6)}-\widehat{\theta}_{n}\end{array}$} \\
\hline & Mean & Stddev & Mean & Stddev & Mean & Stddev \\
\hline \multicolumn{7}{|l|}{ Accelerated Expansion, $\Delta=1 / 52$} \\
\hline$\kappa=0.5$ & 0.239619 & 0.318866 & 0.020498 & 0.017677 & -0.000389 & 0.005771 \\
\hline$\alpha=0.06$ & 0.000362 & 0.017002 & -0.000050 & 0.000536 & 0.000002 & 0.000139 \\
\hline$\sigma=0.15$ & 0.000125 & 0.003367 & 0.000018 & 0.000075 & -0.000000 & 0.000024 \\
\hline \multicolumn{7}{|l|}{ Accelerated Expansion, $\Delta=1 / 12$} \\
\hline$\kappa=0.5$ & 0.050075 & 0.121567 & 0.063831 & 0.104206 & -0.010365 & 0.035368 \\
\hline$\alpha=0.06$ & 0.000036 & 0.008016 & -0.000230 & 0.000230 & 0.000002 & 0.000476 \\
\hline$\sigma=0.15$ & 0.000100 & 0.003429 & 0.000199 & 0.000158 & -0.000027 & 0.000203 \\
\hline \multicolumn{7}{|l|}{ Direct Expansion, $\Delta=1 / 52$} \\
\hline$\kappa=0.5$ & 0.240063 & 0.318818 & 0.047211 & 0.096024 & -0.019166 & 0.198931 \\
\hline$\alpha=0.06$ & 0.000366 & 0.017004 & -0.000015 & 0.000613 & 0.000019 & 0.001251 \\
\hline$\sigma=0.15$ & 0.000124 & 0.003368 & -0.000121 & 0.001457 & 0.000208 & 0.002592 \\
\hline
\end{tabular}




\section{An Alternative Exhibition of the Simulation Results}

The following tables exhibit the Monte Carlo simulation results from Section 6 in an alternative way. Instead of reporting the difference between the approximate MLE and the true MLE, we present the difference between the approximate MLE and the true parameter values, i.e., $\widehat{\theta}_{n}^{(J)}-\widehat{\theta}_{n}$ is replaced by $\widehat{\theta}_{n}^{(J)}-\theta^{\text {True }}$ for each choice of the order $J$. These results show that $\widehat{\theta}_{n}^{(J)}$ inherits the (finite-sample and asymptotic) properties of $\widehat{\theta}_{n}$ for a large enough $J$.

Table 3: Monte Carlo Evidence for the MROU Model

This table exhibits the implementation results for the MROU model. The true parameter values are set as those employed in Section 4. I perform 5000 replications of Monte Carlo simulation of sample path generated by exact Gaussian transition law. Each path contains $n=1000$ observations with weekly $(\Delta=1 / 52)$ or monthly $(\Delta=1 / 12)$ monitoring frequency. The maximum-likelihood estimation is conducted for the true likelihood, the third order approximation $(J=3)$, and the sixth order approximation $(J=6)$.

For finite sample results $(n=1000)$, the mean and standard deviation of the discrepancy between the MLE and the true parameter value (i.e., $\widehat{\theta}_{n}-\theta^{\text {True }}$ ), and the discrepancy between the approximate MLE and the true parameter value (i.e., $\left.\widehat{\theta}_{n}^{(J)}-\theta^{\text {True }}\right)$, for the orders $J=3$ and $J=6$, respectively, are exhibited. Also, I report the asymptotic distribution of $\widehat{\theta}_{n}-\theta^{\text {True }}$ calculated from (6.1) and the Fisher information matrix (6.2).

\begin{tabular}{|c|c|c|c|c|c|c|c|c|}
\hline \multirow[t]{2}{*}{$\begin{array}{c}\text { Parameters } \\
\theta^{\text {True }}\end{array}$} & \multicolumn{2}{|c|}{$\begin{array}{l}\text { Asymptotic } \\
\widehat{\theta}_{n}-\theta^{\text {True }}\end{array}$} & \multicolumn{2}{|c|}{$\begin{array}{c}\text { Finite sample } \\
\widehat{\theta}_{n}-\theta^{\text {True }}\end{array}$} & \multicolumn{2}{|c|}{$\begin{array}{l}\text { Finite sample } \\
\widehat{\theta}_{n}^{(3)}-\theta^{\text {True }}\end{array}$} & \multicolumn{2}{|c|}{$\begin{array}{l}\text { Finite sample } \\
\widehat{\theta}_{n}^{(6)}-\theta^{\text {True }}\end{array}$} \\
\hline & Mean & Stddev & Mean & Stddev & Mean & Stddev & Mean & Stddev \\
\hline \multicolumn{9}{|l|}{$\Delta=1 / 52$} \\
\hline$\kappa=0.5$ & 0 & 0.229136 & 0.245175 & 0.329396 & 0.258653 & 0.341951 & 0.245177 & 0.329402 \\
\hline$\alpha=0.06$ & 0 & 0.013682 & 0.000329 & 0.015202 & 0.000331 & 0.015190 & 0.000329 & 0.015202 \\
\hline$\sigma=0.03$ & 0 & 0.000674 & 0.000021 & 0.000675 & 0.000024 & 0.000676 & 0.000021 & 0.000675 \\
\hline \multicolumn{9}{|l|}{$\Delta=1 / 12$} \\
\hline$\kappa=0.5$ & 0 & 0.111867 & 0.054162 & 0.124773 & 0.083085 & 0.138559 & 0.054159 & 0.124770 \\
\hline$\alpha=0.06$ & 0 & 0.006573 & 0.000097 & 0.006440 & 0.000100 & 0.006430 & 0.000097 & 0.006440 \\
\hline$\sigma=0.03$ & 0 & 0.000685 & 0.000022 & 0.000687 & 0.000047 & 0.000690 & 0.000022 & 0.000687 \\
\hline
\end{tabular}

\section{Two More Examples for Simulation Study}

To further demonstrate the general applicability of our method, I will employ two more sophisticated data-generating processes (arising from financial modeling) with rich drift and diffusion specifications, in which the Lamperti transform either requires computationally demanding implicit integration and inversion or doesn't exist due to a multivariate irreducible specification (see Aït-Sahalia [3] ). It is noteworthy that both of the following two ambitious models violate the technical Assumption 2. Therefore, 


\section{Table 4: Monte Carlo Evidence for the DMROU Model}

This table reports the implementation results for the DMROU model. The true parameter values are set as those employed in Section 4. 1000 replications of Monte Carlo simulation of sample path are generated by exact bivariate Gaussian transition law. Each path contains $n=1000$ observations with daily $(\Delta=1 / 252)$, weekly $(\Delta=1 / 52)$ or monthly $(\Delta=1 / 12)$ monitoring frequency. The maximum-likelihood estimation is conducted for the true likelihood, the second $(J=2)$ and the fourth $(J=4)$ order approximations for the daily case, and the third $(J=3)$ and the sixth $(J=6)$ order approximations for the weekly and monthly cases. For finite sample results $(n=1000)$, the mean and standard deviation of the discrepancy between the MLE and the true parameter value (i.e., $\left.\widehat{\theta}_{n}-\theta^{\text {True }}\right)$, and the discrepancy between the approximate MLE and the true parameter value (i.e., $\widehat{\theta}_{n}^{(J)}-\theta^{\text {True }}$ ), for the orders $J=2,3,4,6$, are exhibited. Also, I report the asymptotic distribution of $\widehat{\theta}_{n}-\theta^{\text {True }}$ calculated from (6.1) and the Fisher information matrix (6.2).

\begin{tabular}{|c|c|c|c|c|c|c|c|c|}
\hline \multirow[t]{2}{*}{$\begin{array}{c}\text { Parameters } \\
\theta^{\text {True }}\end{array}$} & \multicolumn{2}{|c|}{$\begin{array}{l}\text { Asymptotic } \\
\widehat{\theta}_{n}-\theta^{\text {True }}\end{array}$} & \multicolumn{2}{|c|}{$\begin{array}{c}\text { Finite sample } \\
\widehat{\theta}_{n}-\theta^{\text {True }}\end{array}$} & \multicolumn{2}{|c|}{$\begin{array}{l}\text { Finite sample } \\
\widehat{\theta}_{n}^{(2)}-\theta^{\text {True }}\end{array}$} & \multicolumn{2}{|c|}{$\begin{array}{l}\text { Finite sample } \\
\widehat{\theta}_{n}^{(4)}-\theta^{\text {True }}\end{array}$} \\
\hline & Mean & Stddev & Mean & Stddev & Mean & Stddev & Mean & Stddev \\
\hline \multicolumn{9}{|l|}{$\Delta=1 / 252$} \\
\hline$\alpha_{1}=0$ & 0 & 0.100401 & -0.001686 & 0.097716 & -0.000243 & 0.098043 & -0.000977 & 0.098671 \\
\hline$\alpha_{2}=0$ & 0 & 0.051197 & -0.000560 & 0.049661 & -0.000382 & 0.049544 & -0.000491 & 0.049464 \\
\hline$\kappa_{11}=5$ & 0 & 1.587554 & 1.133810 & 2.013933 & 1.138387 & 2.016914 & 1.137202 & 2.012764 \\
\hline$\kappa_{21}=1$ & 0 & 1.602581 & 0.034982 & 2.019396 & 0.024492 & 1.991043 & 0.038172 & 2.020941 \\
\hline$\kappa_{22}=10$ & 0 & 2.240703 & 1.348146 & 2.581314 & 1.350535 & 2.577946 & 1.345664 & 2.577554 \\
\hline \multirow[t]{2}{*}{$\begin{array}{c}\text { Parameters } \\
\theta^{\text {True }}\end{array}$} & \multicolumn{2}{|c|}{$\begin{array}{l}\text { Asymptotic } \\
\widehat{\theta}_{n}-\theta^{\text {True }}\end{array}$} & \multicolumn{2}{|c|}{$\begin{array}{c}\text { Finite sample } \\
\widehat{\theta}_{n}-\theta^{\text {True }}\end{array}$} & \multicolumn{2}{|c|}{$\begin{array}{l}\text { Finite sample } \\
\widehat{\theta}_{n}^{(3)}-\theta^{\text {True }}\end{array}$} & \multicolumn{2}{|c|}{$\begin{array}{l}\text { Finite sample } \\
\widehat{\theta}_{n}^{(6)}-\theta^{\text {True }}\end{array}$} \\
\hline & Mean & Stddev & Mean & Stddev & Mean & Stddev & Mean & Stddev \\
\hline \multicolumn{9}{|l|}{$\Delta=1 / 52$} \\
\hline$\alpha_{1}=0$ & 0 & 0.045625 & -0.000527 & 0.044492 & -0.000433 & 0.044861 & -0.000014 & 0.044320 \\
\hline$\alpha_{2}=0$ & 0 & 0.023290 & -0.000200 & 0.022371 & -0.000175 & 0.022589 & 0.000013 & 0.022477 \\
\hline$\kappa_{11}=5$ & 0 & 0.722188 & 0.247767 & 0.770279 & 0.825690 & 0.944148 & 0.248542 & 0.771369 \\
\hline$\kappa_{21}=1$ & 0 & 0.743389 & -0.012143 & 0.799808 & 0.198778 & 0.972258 & -0.010119 & 0.800769 \\
\hline$\kappa_{22}=10$ & 0 & 1.023690 & 0.266464 & 1.074969 & 2.561354 & 1.575791 & 0.271423 & 1.077062 \\
\hline \multicolumn{9}{|l|}{$\Delta=1 / 12$} \\
\hline$\alpha_{1}=0$ & 0 & 0.022066 & -0.000191 & 0.021364 & -0.000523 & 0.021344 & -0.000122 & 0.021422 \\
\hline$\alpha_{2}=0$ & 0 & 0.011475 & 0.000004 & 0.011109 & -0.000122 & 0.011087 & -0.000101 & 0.011135 \\
\hline$\kappa_{11}=5$ & 0 & 0.355289 & 0.053701 & 0.363768 & 2.735472 & 0.811073 & 0.076233 & 0.370350 \\
\hline$\kappa_{21}=1$ & 0 & 0.398388 & -0.015752 & 0.417193 & 1.341131 & 1.068149 & 0.054105 & 0.449252 \\
\hline$\kappa_{22}=10$ & 0 & 0.534837 & 0.066419 & 0.548926 & 13.589563 & 2.505193 & 0.467701 & 0.651013 \\
\hline
\end{tabular}




\section{Table 5: Monte Carlo Evidence for the SQR Model}

This table shows the implementation results for the SQR model. The true parameter values are set as those employed in Section 4. 5000 replications of Monte Carlo simulation of sample path are generated by exact non-central chi-squared based transition law. Each path contains $n=1000$ observations with weekly $(\Delta=1 / 52)$ or monthly $(\Delta=1 / 12)$ monitoring frequency. The maximumlikelihood estimation is conducted for the true likelihood (expressed via the modified Bessel functions of the first kind), the third order approximation $(J=3)$, and the sixth order approximation $(J=6)$. For finite sample results $(n=1000)$, the mean and standard deviation of the discrepancy between the MLE and the true parameter value (i.e., $\widehat{\theta}_{n}-\theta^{\text {True }}$ ), and the discrepancy between the approximate MLE and the true parameter value (i.e., $\widehat{\theta}_{n}^{(J)}-\theta^{\text {True }}$ ), for the orders $J=3$ and $J=6$, respectively, are exhibited. The accelerated expansion refers to the combination of Lamperti transform and our main expansion procedure, while the direct expansion employs our main expansion procedure bypassing the Lamperti transform.

\begin{tabular}{|c|c|c|c|c|c|c|}
\hline \multirow[t]{2}{*}{$\begin{array}{c}\text { Parameters } \\
\theta^{\text {True }}\end{array}$} & \multicolumn{2}{|c|}{$\begin{array}{c}\text { Finite sample } \\
\widehat{\theta}_{n}-\theta^{\text {True }}\end{array}$} & \multicolumn{2}{|c|}{$\begin{array}{l}\text { Finite sample } \\
\widehat{\theta}_{n}^{(3)}-\theta^{\text {True }}\end{array}$} & \multicolumn{2}{|c|}{$\begin{array}{l}\text { Finite sample } \\
\widehat{\theta}_{n}^{(6)}-\theta^{\text {True }}\end{array}$} \\
\hline & Mean & Stddev & Mean & Stddev & Mean & Stddev \\
\hline \multicolumn{7}{|l|}{ Accelerated Expansion, $\Delta=1 / 52$} \\
\hline$\kappa=0.5$ & 0.239619 & 0.318866 & 0.260117 & 0.332397 & 0.239230 & 0.319120 \\
\hline$\alpha=0.06$ & 0.000362 & 0.017002 & 0.000312 & 0.016959 & 0.000364 & 0.017004 \\
\hline$\sigma=0.15$ & 0.000125 & 0.003367 & 0.000142 & 0.003369 & 0.000124 & 0.003368 \\
\hline \multicolumn{7}{|l|}{ Accelerated Expansion, $\Delta=1 / 12$} \\
\hline$\kappa=0.5$ & 0.050075 & 0.121567 & 0.113907 & 0.170742 & 0.039710 & 0.128845 \\
\hline$\alpha=0.06$ & 0.000036 & 0.008016 & -0.000194 & 0.007997 & 0.000038 & 0.008027 \\
\hline$\sigma=0.15$ & 0.000100 & 0.003429 & 0.000300 & 0.003453 & 0.000074 & 0.003426 \\
\hline \multicolumn{7}{|l|}{ Direct Expansion, $\Delta=1 / 52$} \\
\hline$\kappa=0.5$ & 0.240063 & 0.318818 & 0.287275 & 0.346743 & 0.220897 & 0.384050 \\
\hline$\alpha=0.06$ & 0.000366 & 0.017004 & 0.000350 & 0.016864 & 0.000385 & 0.017095 \\
\hline$\sigma=0.15$ & 0.000124 & 0.003368 & 0.000004 & 0.003675 & 0.000332 & 0.004251 \\
\hline
\end{tabular}


the upcoming demonstration of numerical performance will suggest again the potential applicability of our method to a wide range of diffusion models.

The stochastic elasticity of variance and nonlinear drift model originally proposed in Aït-Sahalia [1] and labeled as SEV-ND (as in Bakshi et al. [4]) is specified as

Model 1. The SEV-ND (stochastic elasticity of variance and nonlinear drift) model:

$$
d X(t)=\left(\alpha_{0}+\alpha_{1} X(t)+\alpha_{2} X(t)^{2}+\alpha_{3} X(t)^{-1}\right) d t+\sqrt{\beta_{0}+\beta_{1} X(t)+\beta_{2} X(t)^{\beta_{3}}} d W(t), \quad X(0)=x_{0} .
$$

The SEV-ND model arose from Aït-Sahalia [1] for modeling spot interest rates and was also investigated in Bakshi et al. [4] for modeling equity volatility. According to the classification in Dai and Singleton [5], the SEV-ND model is non-affine. It integrates a number of desirable features and generalizes a broad set of existing models with features such as mean-reverting, non-linearity of drift, and non-linearity (for example, the constant elasticity feature) of volatility, which allows flexible fit of the volatility smile effect. As pointed out in Bakshi et al. [4], the nonlinear form of volatility results in implicit Lamperti transform and the corresponding inversion, when the method in Aït-Sahalia 2] is applied. To circumvent this difficulty, Bakshi et al. [4] reduced computational efforts to one-dimensional numerical integration for each correction term.

The first several correction terms of our expansion are exhibited as follows:

$$
\begin{aligned}
& \Omega_{0}(y)=\frac{1}{\sqrt{2 \pi}} e^{-\frac{y^{2}}{2}} \\
& \Omega_{1}(y)=\frac{1}{\sqrt{2 \pi}} e^{-\frac{y^{2}}{2}}\left(\frac{y\left(4 \alpha_{0} x_{0}+4 \alpha_{1} x_{0}^{2}+4 \alpha_{2} x_{0}^{3}+4 \alpha_{3}-3 \beta_{1} x_{0}-3 \beta_{2} \beta_{3} x_{0}^{\beta_{3}}\right)}{4 x_{0} \sqrt{\beta_{0}+\beta_{1} x_{0}+\beta_{2} x_{0}^{\beta_{3}}}}+\frac{y^{3}\left(\beta_{1} x_{0}+\beta_{2} \beta_{3} x_{0}^{\beta_{3}}\right)}{4 x_{0} \sqrt{\beta_{0}+\beta_{1} x_{0}+\beta_{2} x_{0}^{\beta_{3}}}}\right), \\
& \Omega_{2}(y)=\frac{1}{\sqrt{2 \pi}} e^{-\frac{y^{2}}{2}}\left(\frac { y ^ { 2 } } { 9 6 x _ { 0 } ^ { 2 } ( \beta _ { 0 } + \beta _ { 1 } x _ { 0 } + \beta _ { 2 } x _ { 0 } ^ { \beta _ { 3 } } ) } \left(48 \alpha_{0}^{2} x_{0}^{2}+96 \alpha_{0} \alpha_{1} x_{0}^{3}+96 \alpha_{0} \alpha_{2} x_{0}^{4}+96 \alpha_{0} \alpha_{3} x_{0}-120 \alpha_{0} \beta_{1} x_{0}^{2}-120 \alpha_{0} \beta_{2} \beta_{3} x_{0}^{\beta_{3}+1}\right.\right. \\
& +48 \alpha_{1}^{2} x_{0}^{4}+96 \alpha_{1} \alpha_{2} x_{0}^{5}+96 \alpha_{1} \alpha_{3} x_{0}^{2}+48 \alpha_{1} \beta_{0} x_{0}^{2}-72 \alpha_{1} \beta_{1} x_{0}^{3}+48 \alpha_{1} \beta_{2} x_{0}^{\beta_{3}+2}-120 \alpha_{1} \beta_{2} \beta_{3} x_{0}^{\beta_{3}+2}+48 \alpha_{2}^{2} x_{0}^{6}+96 \alpha_{2} \alpha_{3} x_{0}^{3} \\
& +96 \alpha_{2} \beta_{0} x_{0}^{3}-24 \alpha_{2} \beta_{1} x_{0}^{4}+96 \alpha_{2} \beta_{2} x_{0}^{\beta_{3}+3}-120 \alpha_{2} \beta_{2} \beta_{3} x_{0}^{\beta_{3}+3}+48 \alpha_{3}^{2}-48 \alpha_{3} \beta_{0}-168 \alpha_{3} \beta_{1} x_{0}-48 \alpha_{3} \beta_{2} x_{0}^{\beta_{3}}-120 \alpha_{3} \beta_{2} \beta_{3} x_{0}^{\beta_{3}} \\
& -36 \beta_{0} \beta_{2} \beta_{3}^{2} x_{0}^{\beta_{3}}+36 \beta_{0} \beta_{2} \beta_{3} x_{0}^{\beta_{3}}+63 \beta_{1}^{2} x_{0}^{2}-36 \beta_{1} \beta_{2} \beta_{3}^{2} x_{0}^{\beta_{3}+1}+162 \beta_{1} \beta_{2} \beta_{3} x_{0}^{\beta_{3}+1}+27 \beta_{2}^{2} \beta_{3}^{2} x_{0}^{2 \beta_{3}}+36 \beta_{2}^{2} \beta_{3} x_{0}^{2 \beta_{3}} \text { ) } \\
& +\frac{1}{96 x_{0}^{2}\left(\beta_{0}+\beta_{1} x_{0}+\beta_{2} x_{0}^{\beta_{3}}\right)}\left(-48 \alpha_{0}^{2} x_{0}^{2}-96 \alpha_{0} \alpha_{1} x_{0}^{3}-96 \alpha_{0} \alpha_{2} x_{0}^{4}-96 \alpha_{0} \alpha_{3} x_{0}+48 \alpha_{0} \beta_{1} x_{0}^{2}+48 \alpha_{0} \beta_{2} \beta_{3} x_{0}^{\beta_{3}+1}-48 \alpha_{1}^{2} x_{0}^{4}\right. \\
& -96 \alpha_{1} \alpha_{2} x_{0}^{5}-96 \alpha_{1} \alpha_{3} x_{0}^{2}-48 \alpha_{1} \beta_{0} x_{0}^{2}-48 \alpha_{1} \beta_{2} x_{0}^{\beta_{3}+2}+48 \alpha_{1} \beta_{2} \beta_{3} x_{0}^{\beta_{3}+2}-48 \alpha_{2}^{2} x_{0}^{6}-96 \alpha_{2} \alpha_{3} x_{0}^{3}-96 \alpha_{2} \beta_{0} x_{0}^{3}-48 \alpha_{2} \beta_{1} x_{0}^{4} \\
& -96 \alpha_{2} \beta_{2} x_{0}^{\beta_{3}+3}+48 \alpha_{2} \beta_{2} \beta_{3} x_{0}^{\beta_{3}+3}-48 \alpha_{3}^{2}+48 \alpha_{3} \beta_{0}+96 \alpha_{3} \beta_{1} x_{0}+48 \alpha_{3} \beta_{2} x_{0}^{\beta_{3}}+48 \alpha_{3} \beta_{2} \beta_{3} x_{0}^{\beta_{3}}+12 \beta_{0} \beta_{2} \beta_{3}^{2} x_{0}^{\beta_{3}} \\
& \left.-12 \beta_{0} \beta_{2} \beta_{3} x_{0}^{\beta_{3}}-9 \beta_{1}^{2} x_{0}^{2}+12 \beta_{1} \beta_{2} \beta_{3}^{2} x_{0}^{\beta_{3}+1}-30 \beta_{1} \beta_{2} \beta_{3} x_{0}^{\beta_{3}+1}+3 \beta_{2}^{2} \beta_{3}^{2} x_{0}^{2 \beta_{3}}-12 \beta_{2}^{2} \beta_{3} x_{0}^{2 \beta_{3}}\right)+\frac{y^{4}}{96 x_{0}^{2}\left(\beta_{0}+\beta_{1} x_{0}+\beta_{2} x_{0}^{\beta_{3}}\right)} \\
& \cdot\left(24 \alpha_{0} \beta_{1} x_{0}^{2}+24 \alpha_{0} \beta_{2} \beta_{3} x_{0}^{\beta_{3}+1}+24 \alpha_{1} \beta_{1} x_{0}^{3}+24 \alpha_{1} \beta_{2} \beta_{3} x_{0}^{\beta_{3}+2}+24 \alpha_{2} \beta_{1} x_{0}^{4}+24 \alpha_{2} \beta_{2} \beta_{3} x_{0}^{\beta_{3}+3}+24 \alpha_{3} \beta_{1} x_{0}+24 \alpha_{3} \beta_{2} \beta_{3} x_{0}^{\beta_{3}}\right. \\
& +8 \beta_{0} \beta_{2} \beta_{3}^{2} x_{0}^{\beta_{3}}-8 \beta_{0} \beta_{2} \beta_{3} x_{0}^{\beta_{3}}-33 \beta_{1}^{2} x_{0}^{2}+8 \beta_{1} \beta_{2} \beta_{3}^{2} x_{0}^{\beta_{3}+1}-74 \beta_{1} \beta_{2} \beta_{3} x_{0}^{\beta_{3}+1}-25 \beta_{2}^{2} \beta_{3}^{2} x_{0}^{2 \beta_{3}}-8 \beta_{2}^{2} \beta_{3} x_{0}^{2 \beta_{3}} \text { ) } \\
& \left.+\frac{y^{6}\left(3 \beta_{1}^{2} x_{0}^{2}+6 \beta_{1} \beta_{2} \beta_{3} x_{0}^{\beta_{3}+1}+3 \beta_{2}^{2} \beta_{3}^{2} x_{0}^{2 \beta_{3}}\right)}{96 x_{0}^{2}\left(\beta_{0}+\beta_{1} x_{0}+\beta_{2} x_{0}^{\beta_{3}}\right)}\right) \text {. }
\end{aligned}
$$

The following model was first introduced in Hagan et al. [6] and labeled as SABR:

Model 2. The SABR (stochastic alpha-beta-rho volatility) model:

$$
\begin{aligned}
d F(t) & =\sigma(t) F(t)^{\beta} d W_{1}(t), F(0)=f_{0}, \\
d \sigma(t) & =\alpha \sigma(t)\left[\rho d W_{1}(t)+\sqrt{1-\rho^{2}} d W_{2}(t)\right], \sigma(0)=\sigma_{0},
\end{aligned}
$$


for $-1 \leq \rho \leq 1,0 \leq \beta \leq 1$ and $\alpha \geq 0$, where $\left\{\left(W_{1}(t), W_{2}(t)\right)\right\}$ is a standard two-dimensional Brownian motion.

It was invented for modeling and pricing fixed-income derivatives. In Model 2, the variable $F$ is usually employed to model forward rates, such as LIBOR forward rates, forward swap rates, or forward stock prices. The evolution of forward $F(t)$ is accompanied with the constant elasticity in $F(t)^{\beta}$ and with the stochastic volatility $\sigma(t)$, which is modeled by a geometric Brownian motion. As a non-affine model (see the classification in Dai and Singleton [5]), the SABR model has a sophisticated specification (such as non-linearity and correlation) of the dispersion matrix

$$
\sigma\left(x_{1}, x_{2}\right)=\left(\begin{array}{cc}
x_{1}^{\beta} x_{2} & 0 \\
\rho \alpha x_{2} & \sqrt{1-\rho^{2}} \alpha x_{2}
\end{array}\right),
$$

which, on the other hand, raises a significant computational challenge. According to the classification in Aït-Sahalia [3], SABR is irreducible. Thus, one is not able to obtain a transformed diffusion with identity dispersion matrix.

The first several correction terms of our expansion are exhibited as follows:

$$
\begin{aligned}
\Omega_{0}\left(y_{1}, y_{2}\right)= & \frac{1}{2 \pi \sqrt{1-\rho^{2}}} e^{\frac{y_{1}^{2}-2 y_{1} y_{2} \rho+y_{2}^{2}}{2\left(\rho^{2}-1\right)}}, \\
\Omega_{1}\left(y_{1}, y_{2}\right)= & \frac{1}{2 \pi} e^{\frac{y_{1}^{2}-2 y_{1} y_{2} \rho+y_{2}^{2}}{2\left(\rho^{2}-1\right)}}\left(\frac{x_{20} y_{1}^{3} \beta x_{10}^{\beta-1}}{2\left(1-\rho^{2}\right)^{3 / 2}}+\frac{y_{1}^{2} y_{2}\left(x_{10} \alpha-x_{20} \beta \rho x_{10}^{\beta}\right)}{2 x_{10}\left(1-\rho^{2}\right)^{3 / 2}}+y_{1}\left(\frac{2 x_{20} \beta \rho^{2} x_{10}^{\beta}-3 x_{20} \beta x_{10}^{\beta}}{2 x_{10}\left(1-\rho^{2}\right)^{3 / 2}}-\frac{y_{2}^{2} \alpha \rho}{\left(1-\rho^{2}\right)^{3 / 2}}\right)\right. \\
& \left.+\frac{y_{2}\left(x_{20} \beta \rho x_{10}^{\beta}+4 x_{10} \alpha \rho^{2}-4 x_{10} \alpha\right)}{2 x_{10}\left(1-\rho^{2}\right)^{3 / 2}}+\frac{y_{2}^{3} \alpha}{2\left(1-\rho^{2}\right)^{3 / 2}}\right),
\end{aligned}
$$

and

$$
\begin{aligned}
& \Omega_{2}\left(y_{1}, y_{2}\right)=\frac{1}{2 \pi} e^{\frac{y_{1}^{2}-2 y_{2} \rho y_{1}+y_{2}^{2}}{2\left(\rho^{2}-1\right)}}\left(\frac{x_{20}^{2} y_{1}^{6} \beta^{2} x_{10}^{2 \beta-2}}{8\left(1-\rho^{2}\right)^{5 / 2}}+y_{1}^{4}\left(\frac{y_{2}^{2}}{24 x_{10}^{2}\left(1-\rho^{2}\right)^{5 / 2}}\left(3 x_{20}^{2} \beta^{2} \rho^{2} x_{10}^{2 \beta}-18 x_{20} \alpha \beta \rho x_{10}^{\beta+1}+3 \alpha^{2} x_{10}^{2}\right)\right.\right. \\
& \left.+\frac{1}{24 x_{10}^{2}\left(1-\rho^{2}\right)^{5 / 2}}\left(-25 x_{20}^{2} \beta^{2} x_{10}^{2 \beta}+19 x_{20}^{2} \beta^{2} \rho^{2} x_{10}^{2 \beta}+4 x_{20}^{2} \beta \rho^{2} x_{10}^{2 \beta}-4 x_{20}^{2} \beta x_{10}^{2 \beta}+\alpha^{2} x_{10}^{2}\right)\right)+y_{1}^{3}\left(\frac{y_{2}^{3}}{24 x_{10}^{2}\left(1-\rho^{2}\right)^{5 / 2}}\right. \\
& \cdot\left(12 x_{20} \alpha \beta \rho^{2} x_{10}^{\beta+1}+6 x_{20} \alpha \beta x_{10}^{\beta+1}-12 \alpha^{2} \rho x_{10}^{2}\right)+\frac{y_{2}}{24 x_{10}^{2}\left(1-\rho^{2}\right)^{5 / 2}}\left(-16 x_{20}^{2} \beta^{2} \rho^{3} x_{10}^{2 \beta}-4 x_{20}^{2} \beta \rho^{3} x_{10}^{2 \beta}+28 x_{20}^{2} \beta^{2} \rho x_{10}^{2 \beta}\right. \\
& \left.\left.+4 x_{20}^{2} \beta \rho x_{10}^{2 \beta}+48 x_{20} \alpha \beta \rho^{2} x_{10}^{\beta+1}-54 x_{20} \alpha \beta x_{10}^{\beta+1}-4 \alpha^{2} \rho x_{10}^{2}\right)\right)+y_{1}\left(-\frac{\alpha^{2} \rho y_{2}^{5}}{2\left(1-\rho^{2}\right)^{5 / 2}}+\frac{y_{2}^{3}}{24 x_{10}^{2}\left(1-\rho^{2}\right)^{5 / 2}}\right. \\
& \cdot\left(-18 x_{20} \alpha \beta x_{10}^{\beta+1}-72 \alpha^{2} \rho^{3} x_{10}^{2}+68 \alpha^{2} \rho x_{10}^{2}\right)+\frac{y_{2}}{24 x_{10}^{2}\left(1-\rho^{2}\right)^{5 / 2}}\left(6 x_{20}^{2} \beta^{2} \rho^{3} x_{10}^{2 \beta}+6 x_{20}^{2} \beta \rho^{3} x_{10}^{2 \beta}-12 x_{20}^{2} \beta^{2} \rho x_{10}^{2 \beta}\right. \\
& \left.\left.-6 x_{20}^{2} \beta \rho x_{10}^{2 \beta}+48 x_{20} \alpha \beta \rho^{4} x_{10}^{\beta+1}-120 x_{20} \alpha \beta \rho^{2} x_{10}^{\beta+1}+72 x_{20} \alpha \beta x_{10}^{\beta+1}-4 \alpha^{2} \rho^{3} x_{10}^{2}+4 \alpha^{2} \rho x_{10}^{2}\right)\right)+y_{1}^{2}\left(\frac{y_{2}^{4}}{24 x_{10}^{2}\left(1-\rho^{2}\right)^{5 / 2}}\right. \\
& \cdot\left(-6 x_{20} \alpha \beta \rho x_{10}^{\beta+1}+6 \alpha^{2} x_{10}^{2}+12 \alpha^{2} \rho^{2} x_{10}^{2}\right)+\frac{y_{2}^{2}}{24 x_{10}^{2}\left(1-\rho^{2}\right)^{5 / 2}}\left(-6 x_{20}^{2} \beta^{2} \rho^{2} x_{10}^{2 \beta}-60 x_{20} \alpha \beta \rho^{3} x_{10}^{\beta+1}+78 x_{20} \alpha \beta \rho x_{10}^{\beta+1}\right. \\
& \left.-34 \alpha^{2} x_{10}^{2}+40 \alpha^{2} \rho^{2} x_{10}^{2}\right)+\frac{1}{24 x_{10}^{2}\left(1-\rho^{2}\right)^{5 / 2}}\left(12 x_{20}^{2} \beta^{2} \rho^{4} x_{10}^{2 \beta}+12 x_{20}^{2} \beta \rho^{4} x_{10}^{2 \beta}+27 x_{20}^{2} \beta^{2} x_{10}^{2 \beta}-36 x_{20}^{2} \beta^{2} \rho^{2} x_{10}^{2 \beta}\right. \\
& \left.\left.-30 x_{20}^{2} \beta \rho^{2} x_{10}^{2 \beta}+18 x_{20}^{2} \beta x_{10}^{2 \beta}-2 \alpha^{2} x_{10}^{2}+2 \alpha^{2} \rho^{2} x_{10}^{2}\right)\right)+\frac{y_{2}^{6} \alpha^{2}}{8\left(1-\rho^{2}\right)^{5 / 2}}+\frac{y_{2}^{4}}{24\left(1-\rho^{2}\right)^{5 / 2} x_{10}^{2}}\left(6 x_{20} \alpha \beta \rho x_{10}^{\beta+1}-35 \alpha^{2} x_{10}^{2}\right. \\
& \left.+36 \alpha^{2} \rho^{2} x_{10}^{2}\right)+\frac{y_{2}^{2}}{24\left(1-\rho^{2}\right)^{5 / 2} x_{10}^{2}}\left(3 x_{20}^{2} \beta^{2} \rho^{2} x_{10}^{2 \beta}+24 x_{20} \alpha \beta \rho^{3} x_{10}^{\beta+1}-24 x_{20} \alpha \beta \rho x_{10}^{\beta+1}+72 \alpha^{2} \rho^{4} x_{10}^{2}+70 \alpha^{2} x_{10}^{2}\right. \\
& \left.-142 \alpha^{2} \rho^{2} x_{10}^{2}\right)+\frac{1}{24\left(1-\rho^{2}\right)^{5 / 2} x_{10}^{2}}\left(6 x_{20}^{2} \beta^{2} \rho^{4} x_{10}^{2 \beta}-6 x_{20}^{2} \beta \rho^{4} x_{10}^{2 \beta}+3 x_{20}^{2} \beta^{2} x_{10}^{2 \beta}-9 x_{20}^{2} \beta^{2} \rho^{2} x_{10}^{2 \beta}+12 x_{20}^{2} \beta \rho^{2} x_{10}^{2 \beta}\right. \\
& \left.\left.-6 x_{20}^{2} \beta x_{10}^{2 \beta}-4 \alpha^{2} \rho^{4} x_{10}^{2}-4 \alpha^{2} x_{10}^{2}+8 \alpha^{2} \rho^{2} x_{10}^{2}\right)+\frac{y_{1}^{5} y_{2}\left(6 x_{10}^{\beta+1} x_{20} \alpha \beta-6 x_{10}^{2 \beta} x_{20}^{2} \beta^{2} \rho\right)}{24\left(1-\rho^{2}\right)^{5 / 2} x_{10}^{2}}\right) \text {. }
\end{aligned}
$$


In the simulation study, sample paths are generated according to the Euler discretization scheme. The mean and standard deviation of the discrepancy between the approximate MLE and the true parameter value (i.e., $\widehat{\theta}_{n}^{(J)}-\theta^{\text {True }}$ ) are reported in Tables [6] and 7 . Though the true likelihood functions are unknown for the SEV-ND and SABR models, the Monte Carlo evidence illustrates that, as the sample size increases while the sampling interval shrinks, the estimation bias of the approximate MLE decreases. It is also seen that a small order of approximation, e.g., $J=3$ for these two models, is enough for delivering estimation with enough accuracy for the monitoring frequency typically encountered in financial modeling. In particular, for the SEV-ND model, daily monitoring cases are tested as an example for relatively high monitoring frequency; for the SABR model, monthly monitoring cases are tested as an example for relatively low monitoring frequency.

Table 6: Monte Carlo Evidence for the SEV-ND Model

Notes. The number of simulation trials is $N=1000$. The true parameter values are set as those estimated for interest rate process employed in Table 4 from Aït-Sahalia 1 .

\begin{tabular}{|c|c|c|c|c|c|c|}
\hline \multirow[t]{2}{*}{$\begin{array}{c}\text { Parameters } \\
\theta^{\text {True }}\end{array}$} & \multicolumn{2}{|c|}{$\begin{array}{c}J=3, n=1000, \Delta=1 / 52 \\
\widehat{\theta}_{n}^{(3)}-\theta^{\text {True }}\end{array}$} & \multicolumn{2}{|c|}{$\begin{array}{c}J=3, n=1000, \Delta=1 / 252 \\
\widehat{\theta}_{n}^{(3)}-\theta^{\text {True }}\end{array}$} & \multicolumn{2}{|c|}{$\begin{array}{c}J=3, n=2000, \Delta=1 / 252 \\
\widehat{\theta}_{n}^{(3)}-\theta^{\text {True }}\end{array}$} \\
\hline & Mean & Stddev & Mean & Stddev & Mean & Stddev \\
\hline$\alpha_{0}=-4.643 \times 10^{-3}$ & 0.000433 & 0.003228 & 0.000169 & 0.000509 & 0.000091 & 0.000365 \\
\hline$\alpha_{1}=4.333 \times 10^{-2}$ & -0.004461 & 0.030525 & -0.001722 & 0.005324 & -0.001002 & 0.003426 \\
\hline$\alpha_{2}=-1.143 \times 10^{-1}$ & -0.011141 & 0.079561 & -0.004391 & 0.007985 & -0.003066 & 0.005080 \\
\hline$\alpha_{3}=1.304 \times 10^{-4}$ & 0.000013 & 0.000131 & 0.000005 & 0.000008 & 0.000003 & 0.000008 \\
\hline$\beta_{0}=1.108 \times 10^{-4}$ & -0.000000 & 0.000009 & -0.000001 & 0.000010 & -0.000000 & 0.000005 \\
\hline$\beta_{1}=-1.883 \times 10^{-3}$ & 0.000002 & 0.000145 & 0.000027 & 0.000127 & 0.000021 & 0.000084 \\
\hline$\beta_{2}=9.681 \times 10^{-3}$ & 0.000179 & 0.001539 & 0.000200 & 0.000753 & 0.000154 & 0.000438 \\
\hline$\beta_{3}=2.073$ & 0.008679 & 0.076845 & 0.019736 & 0.058280 & 0.016767 & 0.040649 \\
\hline
\end{tabular}

\section{Malliavin Calculus and Watanabe-Yoshida Theory: a Brief Survey}

This section provides a brief survey of the Malliavin calculus and the theory of Watanabe [11] and Yoshida [12, 13] to make this paper self-contained. Note that, however, for the purpose of statistical application, one is free to skip these technical details, which enter into the theory only from the verification of convergence, see Theorem 2 .

\subsection{Basic Setup and Notations in Malliavin Calculus}

Malliavin calculus, also known as the stochastic calculus of variations, is an infinite-dimensional differential calculus on the Wiener space. Its novelties were originally tailored to investigate regularity 
Table 7: Monte Carlo Evidence for the SABR Model

Notes. The number of simulation trials is $N=1000$. The true parameter values are set as $\alpha=0.1, \beta=0.999, \rho=-0.3$ without loss of generality for the purpose of illustration.

\begin{tabular}{|c|c|c|c|c|c|c|}
\hline \multirow[t]{2}{*}{$\begin{array}{l}\text { Parameters } \\
\quad \theta^{\text {True }}\end{array}$} & \multicolumn{2}{|c|}{$\begin{array}{c}J=3, n=1000, \Delta=1 / 12 \\
\widehat{\theta}_{n}^{(3)}-\theta^{\text {True }}\end{array}$} & \multicolumn{2}{|c|}{$\begin{array}{c}J=3, n=2000, \Delta=1 / 12 \\
\widehat{\theta}_{n}^{(3)}-\theta^{\text {True }}\end{array}$} & \multicolumn{2}{|c|}{$\begin{array}{c}J=3, n=2000, \Delta=1 / 52 \\
\widehat{\theta}_{n}^{(3)}-\theta^{\text {True }}\end{array}$} \\
\hline & Mean & Stddev & Mean & Stddev & Mean & Stddev \\
\hline$\alpha=0.1$ & -0.000046 & 0.002233 & -0.000139 & 0.002940 & -0.000010 & 0.001547 \\
\hline$\beta=0.999$ & 0.000147 & 0.006839 & 0.000047 & 0.016285 & -0.000186 & 0.003896 \\
\hline$\rho=-0.3$ & -0.001632 & 0.027261 & -0.001591 & 0.020936 & -0.001063 & 0.019224 \\
\hline
\end{tabular}

properties of the law (transition density) of Wiener functional such as solutions of stochastic differential equations. Among many theoretical developments and applications (see, e.g., Nualart [10] and Ikeda and Watanabe [7]), the Malliavin calculus theory established general criteria for a given random vector to possess a smooth density. Instead of making an exhaustive survey of the Malliavin calculus theory, I briefly list some terminologies and notations related to the theory of Watanabe [11] and Yoshida [12, 13].

Let $(\Omega, P, \mathcal{F},\{\mathcal{F}(t)\})$ denote the $d$-dimensional filtered Wiener space, where $\Omega=\mathcal{C}_{0}\left([0, T], \mathbb{R}^{d}\right)$. The coordinate process $\{w(t)\}$ is a $d$-dimensional Brownian motion under the Wiener measure $\mathbb{P}$. Let $H$ be the Cameron-Martin subspace of $\Omega$, i.e.

$$
H=\left\{h=\left(\int_{0}^{\cdot} \dot{h}^{1}(s) d s, \ldots, \int_{0}^{\cdot} \dot{h}^{d}(s) d s\right) ; \quad \dot{h} \in L^{2}[0, T]\right\} .
$$

The inner product of the Hilbert space $H$ is defined as $\left\langle h_{1}, h_{2}\right\rangle_{H}=\sum_{k=1}^{d} \int_{0}^{T} \dot{h}_{1}^{k}(s) \dot{h}_{2}^{k}(s) d s$, for any $h_{1}, h_{2} \in H$. Thus, the norm is equipped with $\|h\|_{H}=\left(\sum_{k=1}^{d} \int_{0}^{T}\left|\dot{h}^{k}(t)\right|^{2} d t\right)^{\frac{1}{2}}$, for $h \in H$. Let $F: \Omega \rightarrow R$ be an $\mathcal{F}(T)$-measurable random variable, which is also called Wiener functional. For $F \in L^{p}(\Omega)$, where $p>1$, we define the directional derivative of $F$ along $h \in H$ as

$$
D_{h} F(w):=\left.\frac{d}{d \epsilon}\right|_{\epsilon=0} F(w+\epsilon h)=\lim _{\epsilon \rightarrow 0} \frac{1}{\epsilon}[F(w+\epsilon h)-F(w)] .
$$

Thus, D.F $(w)$ is defined as a linear functional on Hilbert space $H$. By the Riesz representation theory, there exists an element $D_{s} F(w):=\left(D^{1} F(w), D^{2} F(w), \ldots, D^{d} F(w)\right) \in H$, such that

$$
D_{h} F(w)=\langle\dot{h}, D F(w)\rangle_{H}=\sum_{k=1}^{d} \int_{0}^{T} \dot{h}^{k}(s) D_{s}^{k} F(w) d s .
$$

Let $L^{p}(\Omega: H)$ denote the collection of measurable maps $f$ from $\Omega$ to $H$ such that $\|f\|_{H} \in L^{p}(\Omega)$. If $D F \in L^{p}(\Omega: H), D F$ is defined as the Malliavin derivative of $F$. The analog of Malliavin derivative operator $D$ in finite-dimensional space $\mathbb{R}^{n}$ is the gradient operator. Given a $\mathcal{C}^{1}$-function $f: \mathbb{R}^{n} \rightarrow \mathbb{R}$, the derivative along direction $n$ can be computed as $\frac{\partial f}{\partial n}=\nabla f \cdot n$. This resemblance convinces us 
that the Malliavin calculus generalizes the ordinary finite-dimensional calculus to infinite-dimensional settings. The Malliavin derivative $D F$ can be intuitively regarded as an $H$-valued random variable or a $d$-dimensional stochastic process. Consequently, one is able to define higher order Malliavin derivatives. Let $\mathcal{P}$ denote the collection of polynomials on the Wiener space $\Omega$. Let us define the $s$-times Malliavin norm $\|\cdot\|_{D_{p}^{s}}$ as

$$
\|F\|_{D_{p}^{s}}=\left[\mathbb{E}\|F\|^{p}+\sum_{j=1}^{s} \mathbb{E}\left\|D^{(j)} F\right\|_{H^{\otimes j}}^{p}\right]^{\frac{1}{p}} .
$$

By completing $\mathcal{P}\left(\right.$ in $L^{p}(\Omega)$ ) according to norm $\|\cdot\|_{D_{p}^{s}}$, one constructs a Banach space denoted by $D_{p}^{s}$, which collects all $s$-times Malliavin differentiable variables.

According to standard text of Malliavin calculus, e.g. Nualart [10], for $s \in \mathbb{N}$, the norm (7.2) is equivalent to the one defined as follows. For any Wiener functional $g$, let

$$
\||| g \mid\|_{s, p}=\left\|(I-\mathcal{L})^{\frac{s}{2}} g\right\|_{L^{p}(\Omega)}
$$

where $\mathcal{L}$ is the Ornstein-Uhlenbeck operator. However, using the norm (17.3), one is able to obtain space $D_{p}^{s}$ for any arbitrary real number $s \in \mathbb{R}$ by completing the Wiener polynomial space $\mathcal{P}$. For any $s \in \mathbb{N}$, the equivalence ensures that the completion using norm \|\|$\cdot\|\|_{s, p}$ also generates the space $D_{p}^{s}$. It is also known that the dual space of $D_{p}^{s}$ is $D_{q}^{-s}$, i.e. $\left(D_{p}^{s}\right)_{q}^{\prime-s}$, where $1 / p+1 / q=1$. If $s<0$, the elements in $D_{p}^{s}$ may not be interpreted as ordinary random variables; instead, they are usually regarded as distributions on the Wiener space. If $F \in D_{p}^{s}$ and $G \in D_{q}^{-s}$, let us denote the paring $\langle F, G\rangle$ by $\mathbb{E}(F G)$. Thus, the norm $\|\cdot\|_{-s, q}$ is defined as $\|G\|_{-s, q}=\sup \mathbb{E}(F G)$, where the sup is taken over the set $\left\{F \in D_{p}^{s}:\|F\|_{s, p}<1\right\}$. Now, let us introduce

$$
D^{\infty}:=\bigcap_{p>1} \bigcap_{k>1} D_{p}^{k}, \text { and } D^{-\infty}:=\bigcup_{p>1} \bigcup_{k>1} D_{p}^{-k}
$$

where $D^{\infty}$ is the set of smooth Wiener functionals and $D^{-\infty}$ is the set of generalized Wiener functionals.

The original motivation of the Malliavin calculus theory is to give a probabilistic proof of Hörmander's "sum of squares" theorem (see, e.g., Nualart [10]). The key task in this regard is to establish general criteria in terms of the so called Malliavin covariance matrix for a given random vector to possess a smooth density. In the applications of Malliavin calculus to specific examples, one usually tries to find sufficient conditions for these general criteria to be satisfied. Let $G$ be a $m$-dimensional random variable $G=\left(G^{1}, G^{2}, \ldots, G^{m}\right)$ and denote $D G=\left(D^{j} G^{i}\right)_{m \times d}$, which appears as a $m \times d$ matrix of $H$-valued random variables. The Malliavin covariance matrix is defined as

$$
\Sigma(G)=\left(\Sigma(G)_{i j}\right)_{m \times m},
$$

where

$$
\Sigma(G)_{i j}=\left\langle D G^{i}, D G^{j}\right\rangle_{H}=\sum_{k=1}^{d}\left\langle D^{k} G^{i}, D^{k} G^{j}\right\rangle_{H} .
$$

\subsection{The Watanabe-Yoshida Theory}

The merit of Watanabe 11] begins with a precise and rigorous mathematical interpretation of generalized Wiener functional and its expectation operation. Let $\mathcal{S}$ be the real Schwartz space of rapidly decreasing 
$\mathcal{C}^{\infty}$-functions on $\mathbb{R}^{m}$. Let $\mathcal{S}^{\prime}$ denote the space of Schwartz distribution, which is the dual space of $\mathcal{S}$ and can be regarded as a collection of generalized functions. For $T \in \mathcal{S}^{\prime}$, the variable $T(G)$, for $G \in D^{\infty}$, is interpreted as an element in $D^{-\infty}$. As a linear functional in $D^{-\infty}$, the expectation of $T(G)$ is defined as the paring of $T(G)$ and 1, i.e. $\mathbb{E}[T(G)]=\langle T(G), 1\rangle$. We note that this definition naturally generalizes that of traditional expectation operation for integrable random variables. In particular, by taking $T(z)=\delta(z-x)$ (the Dirac Delta function centered at $x$ ), the expectation $\mathbb{E}[\delta(G-x)]$ represents the density of $G$ evaluated at $x$. This reconciles the following heuristic computation for the density of random variable $G$ :

$$
p_{G}(x)=\frac{\partial}{\partial x} \mathbb{E} 1_{(-\infty, x]}(G)=\mathbb{E}\left[\frac{\partial}{\partial x} 1_{(-\infty, x]}(G)\right]=\mathbb{E}\left[\delta_{x}(G)\right] .
$$

Suppose an $m$-dimensional $D^{\infty}$ random variable $G(\epsilon)$ admits the asymptotic expansion in $D^{\infty}$ : for any $J \in \mathbb{N}$,

$$
G(\epsilon)=\sum_{k=0}^{J} G_{k} \epsilon^{k}+\mathcal{O}\left(\epsilon^{J+1}\right), \quad \text { in } D^{\infty} .
$$

In other words,

$$
\limsup _{\epsilon \rightarrow 0} \frac{1}{\epsilon^{J+1}}\left\|G(\epsilon)-\sum_{k=0}^{J} G_{k} \epsilon^{k}\right\|_{D_{p}^{s}}<+\infty,
$$

for any $p>1, s>0$, where $G_{i} \in D^{\infty}$ for $i=0,1,2,3, \cdots$. For example, Assumption 2 guarantees the Malliavin differentiability of $X^{\epsilon}(1)$ in (3.2) and the convergence of (3.8) holding in $D^{\infty}\left(\mathbb{R}^{m}\right)$, i.e.

$$
\left\|X^{\epsilon}(1)-\sum_{k=0}^{J} F_{k} \epsilon^{k}\right\|_{D_{p}^{s}}=\mathcal{O}\left(\epsilon^{J+1}\right) \text {, for every } p \in(1, \infty) \text { and } s>0 .
$$

Watanabe [1] establishes a condition for the following asymptotic expansion for expectation:

$$
\mathbb{E}[T(G(\epsilon))]=\sum_{k=0}^{J} \mathbb{E} \Phi_{k} \epsilon^{k}+\mathcal{O}\left(\epsilon^{J+1}\right),
$$

where the $\Phi_{k}$ 's are calculated from Taylor-like expansion of $T(G(\epsilon))$ with respect to $\epsilon$. To obtain (7.7), it is sufficient to establish the following expansion,

$$
T(G(\epsilon))=\sum_{k=0}^{J} \Phi_{k} \epsilon^{k}+\mathcal{O}\left(\epsilon^{J+1}\right), \quad \text { in } D^{-\infty} .
$$

In other words, for all $p>1$ and some $s>0$,

$$
\limsup _{\epsilon \rightarrow 0} \frac{1}{\epsilon^{J+1}}\left\|T(G(\epsilon))-\sum_{k=0}^{J} \Phi_{k} \epsilon^{k}\right\|_{D_{p}^{-s}}<+\infty,
$$

where $\Phi_{i} \in D_{p}^{-s}$, for $i=0,1,2,3, \cdots$. According to Theorem 2.3 in Watanabe 11], it is justified that the asymptotic expansions in (7.8) and thus in (7.7) are valid if the Malliavin covariance of $G(\epsilon)$ is uniformly non-degenerated in the sense that

$$
\limsup _{\epsilon \rightarrow 0} \mathbb{E}\left[\operatorname{det}(\Sigma(G(\epsilon)))^{-p}\right]<\infty, \quad \text { for all } p \in(0,+\infty) .
$$


Therefore, a crucial step for applying the Watanabe theory is the verification of the non-degeneracy of the Malliavin covariance (7.9). In general, the verification of the uniform non-degenerate condition (7.9) is not easy even for some simple cases, where the underlying Malliavin covariance is expressed by an integration of some adaptive processes. However, it has been proved in Watanabe [11] that, for $X^{\epsilon}(1)$ as introduced in (3.2), the standardized variable $Y^{\epsilon}(1)=D\left(x_{0}\right)\left(X^{\epsilon}(1)-x_{0}\right) / \epsilon$ is uniformly nondegenerate if and only if the diffusion matrix $A\left(x_{0}\right)=\sigma\left(x_{0}\right) \sigma\left(x_{0}\right)^{T}$ is non-singular, i.e. $\operatorname{det} A\left(x_{0}\right)>0$. Motivated by the statistical estimation of continuously monitored "small diffusions", Yoshida 12, 13] established a truncated version of the asymptotic expansion theory, in which verification of the uniform non-degeneracy of Malliavin covariance became easier.

\subsection{Uniform Convergence of the Asymptotic Expansion}

In order to apply the Watanabe-Yoshida theory to our approximate maximum-likelihood estimation, I set up the following lemma for incorporating uniform convergence in various parameters. The expansions (7.5), (7.7) and (7.8) (or equivalently those in Watanabe [1] and Yoshida [13]), can be directly adapted to a version addressing uniform convergence as follows. The proof is a generalization of Theorem 2.3 in Watanabe [1]] or Theorem 2.2 of Yoshida [13], and thus is omitted.

LEMMA 1. Assume that $G(\epsilon, \vartheta) \in D^{\infty}\left(\mathbb{R}^{m}\right), \epsilon \in(0,1], \vartheta \in \Upsilon$, where $\Upsilon$ denotes a set of parameters, is uniformly non-degenerate in the sense of (7.9) and has an asymptotic expansion in $D^{\infty}$, i.e., for any $J \in \mathbb{N}$,

$$
G(\epsilon, \vartheta)=\sum_{k=0}^{J} G_{k}(\vartheta) \epsilon^{k}+\mathcal{O}\left(\epsilon^{J+1}\right), \text { as } \epsilon \rightarrow 0,
$$

uniformly in $\vartheta \in \Upsilon$. Here, $G_{k}(\vartheta)=\left(G_{k, 1}(\vartheta), G_{k, 2}(\vartheta), \cdots, G_{k, m}(\vartheta)\right)$. Let the index set $\Lambda$ be a Borel $\sigma$-field of $\mathbb{R}^{m}$ and $\left\{T_{\lambda} ; \lambda \in \Lambda\right\} \subset \mathcal{S}^{\prime}\left(\mathbb{R}^{m}\right)$. Then, $T_{\lambda}(G(\epsilon, \vartheta))$ has an asymptotic expansion in $D^{-\infty}$ :

$$
T_{\lambda}[G(\epsilon, \vartheta)]=\sum_{k=0}^{J} \Phi_{k, \vartheta}(\lambda) \epsilon^{k}+\mathcal{O}\left(\epsilon^{J+1}\right), \text { as } \epsilon \rightarrow 0 .
$$

This expansion is uniform in $\lambda \in \Lambda$ and $\vartheta \in \Upsilon$. The $k$-th expansion term $\Phi_{k, \vartheta}(\lambda)$ is determined by

$$
\Phi_{k, \vartheta}(\lambda)=\sum_{(l, \mathbf{i}(l), \mathbf{j}(l)) \in S_{k}} \frac{1}{l(\mathbf{i}) !} \frac{\partial}{\partial x_{i_{1}}} \frac{\partial}{\partial x_{i_{2}}} \cdots \frac{\partial}{\partial x_{i_{l}}} T_{\lambda}\left(G_{0}(\vartheta)\right) G_{j_{1}, i_{1}}(\vartheta) G_{j_{2}, i_{2}}(\vartheta) \cdots G_{j_{l}, i_{l}}(\vartheta),
$$

where the index set $S_{k}$ is defined in (3.23). For $l=0$, one regards $\frac{\partial}{\partial x_{i_{1}}} \frac{\partial}{\partial x_{i_{2}}} \cdots \frac{\partial}{\partial x_{i_{l}}}$ as an identity 
mapping. In particular, the first several expansion terms are explicitly calculated as

$$
\begin{aligned}
\Phi_{1, \vartheta}(\lambda)= & \sum_{i=1}^{m} G_{1, i}(\vartheta) \frac{\partial T_{\lambda}\left(G_{0}(\vartheta)\right)}{\partial x_{i}}, \\
\Phi_{2, \vartheta}(\lambda)= & \sum_{i=1}^{m} G_{2, i}(\vartheta) \frac{\partial T_{\lambda}\left(G_{0}(\vartheta)\right)}{\partial x_{i}}+\frac{1}{2 !} \sum_{i, j=1}^{m} G_{1, i}(\vartheta) G_{1, j}(\vartheta) \frac{\partial^{2} T_{\lambda}\left(G_{0}(\vartheta)\right)}{\partial x_{i} \partial x_{j}} \\
\Phi_{3, \vartheta}(\lambda)= & \sum_{i=1}^{m} G_{3, i}(\vartheta) \frac{\partial T_{\lambda}\left(G_{0}(\vartheta)\right)}{\partial x_{i}}+\frac{2}{2 !} \sum_{i, j=1}^{m} G_{1, i}(\vartheta) G_{2, j}(\vartheta) \frac{\partial^{2} T_{\lambda}\left(G_{0}(\vartheta)\right)}{\partial x_{i} \partial x_{j}} \\
& +\frac{1}{3 !} \sum_{i, j, k=1}^{m} G_{1, i}(\vartheta) G_{1, j}(\vartheta) G_{1, k}(\vartheta) \frac{\partial^{3} T_{\lambda}\left(G_{0}(\vartheta)\right)}{\partial x_{i} \partial x_{j} \partial x_{k}} .
\end{aligned}
$$

As a result, one obtains the asymptotic expansion of the expectation:

$$
\mathbb{E} T_{\lambda}[G(\epsilon, \vartheta)]=\sum_{k=0}^{J} \mathbb{E} \Phi_{k, \vartheta}(\lambda) \epsilon^{k}+\mathcal{O}\left(\epsilon^{J+1}\right) \text {, as } \epsilon \rightarrow 0 .
$$

\section{Proof of Lemma 2}

To prove Lemma 2, we begin with the following lemma.

LEMMA 2. Under Assumption 2, for any $0<t<\infty, p>1$ and $j \geq 0$, one has that $\partial^{(j)} X^{\epsilon}(t) / \partial \epsilon^{j} \in$ $L^{p}(\Omega)$, uniformly in $\left(x_{0}, \theta\right) \in K \times \Theta$ and $0<\epsilon<1$.

Proof. Employing a standard argument for analyzing stochastic differential equations (see, e.g., Chapter 2 from Nualart [10]), I prove the result by induction. First, let us consider the case of $j=0$. Without loss of generality, I prove that $X^{\epsilon}(t) \in L^{p}(\Omega)$, for any $p>1$ uniformly in $\left(x_{0}, \theta\right) \in K \times \Theta$ and $0<\epsilon<1$, for the case of $m=1$. The multidimensional case is a straightforward extension.

Recall that

$$
X^{\epsilon}(t)=x_{0}+\epsilon^{2} \int_{0}^{t} \mu\left(X^{\epsilon}(s) ; \theta\right) d s+\epsilon \int_{0}^{t} \sigma\left(X^{\epsilon}(s) ; \theta\right) d W(s) .
$$

Therefore, it follows that

$$
\mathbb{E}\left|X^{\epsilon}(t)\right|^{p} \leq 3^{p-1}\left(\left|x_{0}\right|^{p}+\epsilon^{2 p} \mathbb{E}\left|\int_{0}^{t} \mu\left(X^{\epsilon}(s) ; \theta\right) d s\right|^{p}+\epsilon^{p} \mathbb{E}\left|\int_{0}^{t} \sigma\left(X^{\epsilon}(s) ; \theta\right) d W(s)\right|^{p}\right) .
$$

According to the assumptions (A.3) and (A.4), one obtains that

$$
\begin{aligned}
\left|\mu\left(X^{\epsilon}(s) ; \theta\right)\right| & \leq \mu_{1}\left|X^{\epsilon}(s)-x_{0}\right|+\mu_{0} \leq \mu_{1}\left|X^{\epsilon}(s)\right|+\mu_{1}\left|x_{0}\right|+\mu_{0}, \\
\left|\sigma\left(X^{\epsilon}(s) ; \theta\right)\right| & \leq \sigma_{1}\left|X^{\epsilon}(s)-x_{0}\right|+\sigma_{0} \leq \sigma_{1}\left|X^{\epsilon}(s)\right|+\sigma_{1}\left|x_{0}\right|+\sigma_{0} .
\end{aligned}
$$

Using the Hölder inequality, one has that

$$
\begin{aligned}
\mathbb{E}\left|\int_{0}^{t} \mu\left(X^{\epsilon}(s) ; \theta\right) d s\right|^{p} & \leq \mathbb{E}\left(\int_{0}^{t}\left|\mu\left(X^{\epsilon}(s) ; \theta\right)\right| d s\right)^{p} \leq t^{p-1} \mathbb{E}\left(\int_{0}^{t}\left|\mu\left(X^{\epsilon}(s) ; \theta\right)\right|^{p} d s\right) \\
& \leq 2^{p-1} t^{p-1} \mathbb{E} \int_{0}^{t}\left[\mu_{1}^{p}\left|X^{\epsilon}(s)\right|^{p}+\left(\mu_{1}\left|x_{0}\right|+\mu_{0}\right)^{p}\right] d s \\
& \leq 2^{p-1} t^{p-1} \mu_{1}^{p} \mathbb{E} \int_{0}^{t}\left|X^{\epsilon}(s)\right|^{p} d s+2^{p-1} t^{p}\left(\mu_{1}\left|x_{0}\right|+\mu_{0}\right)^{p} .
\end{aligned}
$$


For the local martingale $M(t)=\int_{0}^{t} \sigma\left(X^{\epsilon}(s) ; \theta\right) d W(s)$, direct application of the Burkholder-Gundy inequality (see, e.g., p. 166 in Karatzas and Shreve [8]) yields that

$$
\begin{aligned}
\mathbb{E}|M(t)|^{p} & =\mathbb{E}\left|\int_{0}^{t} \sigma\left(X^{\epsilon}(s) ; \theta\right) d W(s)\right|^{p} \\
& \leq c_{1}(p) \mathbb{E}\left[\langle M\rangle_{t}\right]^{\frac{p}{2}}=c_{1}(p) \mathbb{E}\left(\int_{0}^{t}\left[\sigma\left(X^{\epsilon}(s) ; \theta\right)\right]^{2} d s\right)^{\frac{p}{2}},
\end{aligned}
$$

for any $p \geq 2$. By the Hölder inequality, it follows that

$$
\begin{aligned}
\mathbb{E}\left(\int_{0}^{t}\left[\sigma\left(X^{\epsilon}(s) ; \theta\right)\right]^{2} d s\right)^{\frac{p}{2}} & \leq t^{\frac{p}{2}-1} \mathbb{E}\left(\int_{0}^{t}\left[\sigma\left(X^{\epsilon}(s) ; \theta\right)\right]^{p} d s\right) \\
& \leq 2^{p-1} t^{\frac{p}{2}-1} \mathbb{E} \int_{0}^{t}\left[\sigma_{1}^{p}\left|X^{\epsilon}(s)\right|^{p}+\left(\sigma_{1}\left|x_{0}\right|+\sigma_{0}\right)^{p}\right] d s \\
& \leq 2^{p-1} t^{\frac{p}{2}-1} \sigma_{1}^{p} \mathbb{E} \int_{0}^{t}\left|X^{\epsilon}(s)\right|^{p} d s+2^{p-1} t^{\frac{p}{2}}\left(\sigma_{1}\left|x_{0}\right|+\sigma_{0}\right)^{p}
\end{aligned}
$$

Thus, the following inequality is established

$$
\mathbb{E}\left|X^{\epsilon}(t)\right|^{p} \leq \alpha\left(T, x_{0}, \mu_{0}, \mu_{1}, \sigma_{1}, \sigma_{0}\right)+\beta\left(T, \mu_{0}, \mu_{1}, \sigma_{1}, \sigma_{0}\right) \int_{0}^{t} \mathbb{E}\left|X^{\epsilon}(s)\right|^{p} d s
$$

for any $T>0$ and $t \in[0, T]$, where the coefficients are defined as

$$
\begin{aligned}
\alpha\left(T, x_{0}, \mu_{0}, \mu_{1}, \sigma_{1}, \sigma_{0}\right)= & 3^{p-1}\left|x_{0}\right|^{p}+3^{p-1} \epsilon^{2 p} 2^{p-1} t^{p}\left(\mu_{1}\left|x_{0}\right|+\mu_{0}\right)^{p} \\
& +3^{p-1} \epsilon^{p} c_{1}(p) 2^{p-1} t^{\frac{p}{2}}\left(\sigma_{1}\left|x_{0}\right|+\sigma_{0}\right)^{p}, \\
\beta\left(T, \mu_{0}, \mu_{1}, \sigma_{1}, \sigma_{0}\right)= & 3^{p-1} \epsilon^{2 p} 2^{p-1} t^{p-1} \mu_{1}^{p}+3^{p-1} \epsilon^{p} c_{1}(p) 2^{p-1} t^{\frac{p}{2}-1} \sigma_{1}^{p} .
\end{aligned}
$$

By the Gronwall inequality, we obtain that

$$
\mathbb{E}\left|X^{\epsilon}(t)\right|^{p} \leq \alpha\left(T, x_{0}, \mu_{0}, \mu_{1}, \sigma_{1}, \sigma_{0}\right) e^{\beta\left(T, \mu_{0}, \mu_{1}, \sigma_{1}, \sigma_{0}\right) t}
$$

Since $\alpha$ and $\beta$ are uniformly bounded in $x_{0} \in K$, we have that $X^{\epsilon}(t) \in L^{p}(\Omega)$, for any $p \geq 2$ uniformly in $\left(x_{0}, \theta\right) \in K \times \Theta$. Hence, we have that $X^{\epsilon}(t) \in L^{p}(\Omega)$ for any $p>1$.

Assuming that $\frac{\partial^{(k)} X^{\epsilon}(t)}{\partial \epsilon^{k}} \in L^{p}(\Omega)$ for any $k=0,1, \cdots, j$, I verify $\frac{\partial^{(j+1)} X^{\epsilon}(t)}{\partial \epsilon^{j+1}} \in L^{p}(\Omega)$ by induction. Indeed, inheriting the idea of stochastic flow (see, e.g., Chapter V in Ikeda and Watanabe 7]), we obtain the following SDE governing $\frac{\partial^{(j+1)} X^{\epsilon}(t)}{\partial \epsilon^{j+1}}$ :

$$
\begin{aligned}
d\left(\frac{\partial^{(j+1)} X^{\epsilon}(t)}{\partial \epsilon^{j+1}}\right)= & \left(a_{j+1}(t, X, \epsilon ; \theta)+\epsilon^{2} \mu^{\prime}\left(X^{\epsilon}(t) ; \theta\right) \frac{\partial^{(j+1)} X^{\epsilon}(t)}{\partial \epsilon^{j+1}}\right) d t \\
& +\left(b_{j+1}(t, X, \epsilon ; \theta)+\epsilon \sigma^{\prime}\left(X^{\epsilon}(t) ; \theta\right) \frac{\partial^{(j+1)} X^{\epsilon}(t)}{\partial \epsilon^{j+1}}\right) d W(t),
\end{aligned}
$$


where the coefficients are

$$
\begin{aligned}
& a_{j+1}(t, X, \epsilon, \theta)=f_{j+1}\left(\epsilon, \mu, \mu^{\prime}, \cdots, \mu^{(j)} ; X^{\epsilon}(t), \frac{\partial X^{\epsilon}(t)}{\partial \epsilon} \ldots, \frac{\partial^{(j)} X^{\epsilon}(t)}{\partial \epsilon^{j}}\right), \\
& b_{j+1}(t, X, \epsilon, \theta)=g_{j+1}\left(\epsilon, \sigma, \sigma^{\prime}, \cdots, \sigma^{(j)} ; X^{\epsilon}(t), \frac{\partial X^{\epsilon}(t)}{\partial \epsilon} \ldots, \frac{\partial^{(j)} X^{\epsilon}(t)}{\partial \epsilon^{j}}\right) .
\end{aligned}
$$

for some smooth functions $f_{j+1}$ and $g_{j+1}$. By the induction assumption, it is obvious that

$$
a_{j+1}(t, X, \epsilon, \theta), b_{j+1}(t, X, \epsilon, \theta) \in L^{p}(\Omega) \text {, for any } k=0,1, \cdots, j .
$$

Thus, by solving $\frac{\partial^{(j+1)} X^{\epsilon}(t)}{\partial \epsilon^{j+1}}$ explicitly, we have that

$$
\begin{aligned}
\frac{\partial^{(j+1)} X^{\epsilon}(t)}{\partial \epsilon^{j+1}}= & \Lambda(t)\left\{\int_{0}^{t} \Lambda(u)^{-1}\left[a_{j+1}(u, X, \epsilon ; \theta)-\epsilon \sigma^{\prime}\left(X^{\epsilon}(u) ; \theta\right) b_{j+1}(u, X, \epsilon ; \theta)\right] d u\right. \\
& \left.+\int_{0}^{t} \Lambda(u)^{-1} b_{j+1}(u, X, \epsilon ; \theta) d W(u)\right\},
\end{aligned}
$$

where

$$
\Lambda(t)=\exp \left(\int_{0}^{t} \epsilon^{2} \mu^{\prime}\left(X^{\epsilon}(u) ; \theta\right) d u+\int_{0}^{t} \epsilon \sigma^{\prime}\left(X^{\epsilon}(u) ; \theta\right) d W(u)-\frac{1}{2} \int_{0}^{t}\left[\epsilon \sigma^{\prime}\left(X^{\epsilon}(u) ; \theta\right)\right]^{2} d u\right) .
$$

The similar inequality arguments, as what is done in the induction foundation, yield that $\frac{\partial^{(j+1)} X^{\epsilon}(t)}{\partial \epsilon^{j+1}} \in$ $L^{p}(\Omega)$, for any $0<t<1, p>1$, uniformly in $\left(x_{0}, \theta\right) \in K \times \Theta$ and $0<\epsilon<1$.

Now, we prove Lemma 2.

Proof. The proof of this lemma follows from the similar lines of argument for the Theorem 7.1 in Malliavin and Thalmaier 9]. Without loss of generality, I begin with the proof of

$$
\left\|Y^{\epsilon}\left(\theta, x_{0}\right)-\left.\sum_{k=0}^{J} \frac{1}{k !} \frac{\partial^{(k)} Y^{\epsilon}\left(\theta, x_{0}\right)}{\partial \epsilon^{k}}\right|_{\epsilon=0} \epsilon^{k}\right\|_{L^{p}}=\mathcal{O}\left(\epsilon^{J+1}\right) .
$$

Employing the integral remainder form of Taylor expansion, one obtains that

$$
\begin{aligned}
& X^{\epsilon}\left(1 ; \theta, x_{0}\right)-\left.\sum_{k=0}^{J+1} \frac{1}{k !} \frac{\partial^{(k)} X^{\epsilon}\left(1 ; \theta, x_{0}\right)}{\partial \epsilon^{k}}\right|_{\epsilon=0} \epsilon^{k} \\
= & \left.\int_{0}^{\epsilon} \int_{0}^{s_{n+2}} \cdots \int_{0}^{s_{2}} \frac{\partial^{(J+2)} X^{\epsilon}\left(1 ; \theta, x_{0}\right)}{\partial \epsilon^{J+2}}\right|_{\epsilon=s_{1}} d s_{1} d s_{2} \cdots d s_{n+2} .
\end{aligned}
$$

Thus, by Lemma 2, it yields that

$$
\begin{aligned}
& \left\|X^{\epsilon}\left(1 ; \theta, x_{0}\right)-\left.\sum_{k=0}^{J+1} \frac{1}{k !} \frac{\partial^{(k)} X^{\epsilon}\left(1 ; \theta, x_{0}\right)}{\partial \epsilon^{k}}\right|_{\epsilon=0} \epsilon^{k}\right\|_{L^{p}} \\
= & \epsilon^{J+2}\left\|\left.\int_{0}^{1} \int_{0}^{r_{n+2}} \cdots \int_{0}^{r_{2}} \frac{\partial^{(J+2)} X^{\epsilon}\left(1 ; \theta, x_{0}\right)}{\partial \epsilon^{J+2}}\right|_{\epsilon=\epsilon r_{1}} d r_{1} d r_{2} \cdots d r_{n+2}\right\|_{L^{p}}=\mathcal{O}\left(\epsilon^{J+2}\right),
\end{aligned}
$$


uniformly in $\left(x_{0}, \theta\right) \in K \times \Theta$. Similarly, we obtain that

$$
\left\|D^{j} X^{\epsilon}\left(1 ; \theta, x_{0}\right)-\left.\sum_{k=0}^{J+1} \frac{1}{k !} \frac{\partial^{(k)}}{\partial \epsilon^{k}} D^{j} X^{\epsilon}\left(1 ; \theta, x_{0}\right)\right|_{\epsilon=0} \epsilon^{k}\right\|_{L^{p}(H \otimes \cdots \otimes H)}=\mathcal{O}\left(\epsilon^{J+2}\right) .
$$

Because

$$
\left.D^{j}\left(\frac{1}{k !} \frac{\partial^{(k)} X^{\epsilon}\left(1 ; \theta, x_{0}\right)}{\partial \epsilon^{k}}\right)\right|_{\epsilon=0}=\left.\frac{1}{k !} \frac{\partial^{(k)}}{\partial \epsilon^{k}} D^{j} X^{\epsilon}\left(1 ; \theta, x_{0}\right)\right|_{\epsilon=0},
$$

we have that

$$
\left\|D^{j} X^{\epsilon}\left(1 ; \theta, x_{0}\right)-\left.\sum_{k=0}^{J+1} D^{j}\left(\frac{1}{k !} \frac{\partial^{(k)} X^{\epsilon}\left(1 ; \theta, x_{0}\right)}{\partial \epsilon^{k}}\right)\right|_{\epsilon=0} \epsilon^{k}\right\|_{L^{p}(H \otimes \cdots \otimes H)}=\mathcal{O}\left(\epsilon^{J+2}\right),
$$

see, e.g. standard arguments in Chapter 2 of Nualart [10]. Similar but tedious arguments apply to higher order Malliavin derivatives. Therefore, it follows that

$$
\left\|X^{\epsilon}\left(1 ; \theta, x_{0}\right)-\left.\sum_{k=0}^{J+1} \frac{1}{k !} \frac{\partial^{(k)} X^{\epsilon}\left(1 ; \theta, x_{0}\right)}{\partial \epsilon^{k}}\right|_{\epsilon=0} \epsilon^{k}\right\|_{D_{p}^{s}}=\mathcal{O}\left(\epsilon^{J+2}\right) .
$$

By the standardization (3.16), we obtain that

$$
\left\|Y^{\epsilon}\left(\theta, x_{0}\right)-\left.\sum_{k=0}^{J} \frac{1}{k !} \frac{\partial^{(k)} Y^{\epsilon}\left(\theta, x_{0}\right)}{\partial \epsilon^{k}}\right|_{\epsilon=0} \epsilon^{k}\right\|_{D_{p}^{s}}=\mathcal{O}\left(\epsilon^{J+1}\right),
$$

uniformly in $\left(x_{0}, \theta\right) \in K \times \Theta$ for any $p \geq 1$ and $s \in \mathbb{N}$.

\section{References}

[1] Aїт-Sahalia, Y. (1996). Testing continuous-time models of the spot interest rate. Review of Financial Studies, 9 385-426.

[2] AïT-Sahalia, Y. (2002). Maximum-likelihood estimation of discretely-sampled diffusions: A closed-form approximation approach. Econometrica, $70223-262$.

[3] Aït-Sahalia, Y. (2008). Closed-form likelihood expansions for multivariate diffusions. Annals of Statistics, 36 906-937.

[4] Bakshi, G., Ju, N. and Ou-Yang, H. (2006). Estimation of continuous-time models with an application to equity volatility dynamics. Journal of Financial Economics, 82 227-249.

[5] Dai, Q. and Singleton, K. J. (2000). Specification analysis of affine term structure models. Journal of Finance, 55 1943-1978.

[6] Hagan, P. D., A.S.Lesniewski and D.E.Woodward (2002). Managing smile risk. Wilmott Magazine Separtember 84-108. 
[7] Ikeda, N. and Watanabe, S. (1989). Stochastic Differential Equations and Diffusion Processes. 2nd ed. North-Holland Mathematical Library, North-Holland.

[8] Karatzas, I. and Shreve, S. E. (1991). Brownian Motion and Stochastic Calculus, vol. 113 of Graduate Texts in Mathematics. 2nd ed. Springer-Verlag, New York.

[9] Malliavin, P. and Thalmaier, A. (2006). Stochastic Calculus of Variations in Mathematical Finance. Springer Finance. Berlin: Springer.

[10] Nualart, D. (2006). The Malliavin Calculus and Related Topics. 2nd ed. Probability and Its Applications. Berlin: Springer.

[11] Watanabe, S. (1987). Analysis of Wiener functionals (Malliavin calculus) and its applications to heat kernels. Annals of Probability, 15 1-39.

[12] Yoshida, N. (1992). Asymptotic expansions for statistics related to small diffusions. Journal of Japan Statistical Society, 22 139-159.

[13] Yoshida, N. (1992). Asymptotic expansions of maximum likelihood estimators for small diffusions via the theory of Malliavin-Watanabe . Probability Theory and Related Fields, 92 275-311. 